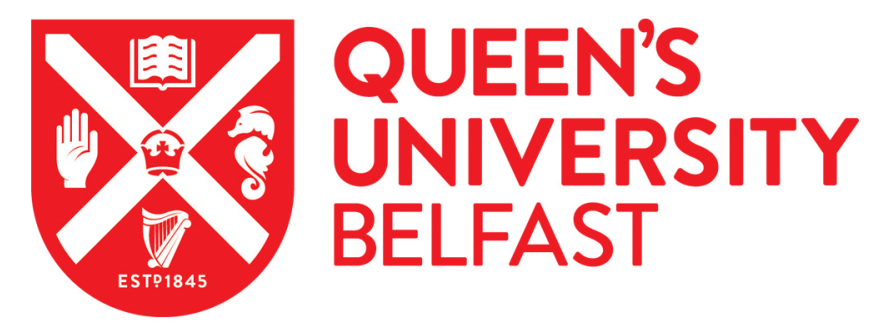

\title{
Formulation of Locally Reacting Surfaces in FDTD/K-DWM Modelling of Acoustic Spaces
}

Kowalczyk, K., \& Van Walstijn, M. (2008). Formulation of Locally Reacting Surfaces in FDTD/K-DWM Modelling of Acoustic Spaces. Acta Acustica united with Acustica, 94(6), 891-906. https://doi.org/10.3813/AAA.918107

Published in:

Acta Acustica united with Acustica

Document Version:

Peer reviewed version

Queen's University Belfast - Research Portal:

Link to publication record in Queen's University Belfast Research Portal

\section{General rights}

Copyright for the publications made accessible via the Queen's University Belfast Research Portal is retained by the author(s) and / or other copyright owners and it is a condition of accessing these publications that users recognise and abide by the legal requirements associated with these rights.

Take down policy

The Research Portal is Queen's institutional repository that provides access to Queen's research output. Every effort has been made to ensure that content in the Research Portal does not infringe any person's rights, or applicable UK laws. If you discover content in the Research Portal that you believe breaches copyright or violates any law, please contact openaccess@qub.ac.uk. 


\section{Formulation of Locally Reacting Surfaces in FDTD/K-DWM Modelling of Acoustic Spaces}

\author{
Konrad Kowalczyk, Maarten van Walstijn \\ Sonic Arts Research Centre (SARC), School of \\ Electronics, Electrical Engineering and Computer Science, \\ Queen's University, Belfast BT7 1NN, Northern Ireland, \\ UK \\ <kkowalczyk01@qub.ac.uk>, \\ $<$ m.vanwalstijn@qub.ac.uk>
}

Short title: FDTD Formulation of Locally Reacting Surfaces

\section{Summary}

In this paper, we present new methods for constructing and analysing formulations of locally reacting surfaces that can be used in finite difference time domain (FDTD) simulations of acoustic spaces. Novel FDTD formulations of frequency-independent and frequency-dependent impedance boundaries are proposed for 2D and 3D acoustic systems, including a full treatment of corners and boundary edges. The proposed boundary formulations are designed for virtual acoustics applications using the standard leapfrog scheme based on the rectilinear grid, and apply to FDTD as well as Kirchhoff variable digital waveguide mesh (K-DWM) methods. In addition, new analytic evaluation methods that accurately predict the reflectance of numerical boundary formulations are proposed.

The results obtained from numerical experiments and numerical boundary analysis (NBA) are analysed in time and frequency domains in terms of the pressure wave reflectance for different angles of incidence and various impedances. The results show that the proposed boundary formulations structurally adhere well to the theoretical reflectance. In particular, both reflectance magnitude and phase are closely approximated even at high angles of incidence and low impedances. Furthermore, excellent agreement was found between the numerical boundary analysis and the experimental results, validating both as tools for researching FDTD boundary formulations.

PACS number: 43.55.Ka

\section{Introduction}

Research on numerical simulation of acoustic spaces is dominated by two distinct approaches, namely geometrical techniques, such as ray tracing or the image source method, and wave-based methods. The former are relatively efficient in computation but not entirely physical in their formulation, and therefore limited in terms of predictive capacity. This limitation is generally apparent for low and middle frequency ranges, and particularly so when applied to modelling small enclosures or rooms with highly non-rigrid walls $[1,2]$. Wave-based methods on the other hand simulate the acoustical equations directly and therefore have the advantage of inherently modelling wave-related phenomena such as diffraction, be it that the computational costs for wideband applications are high, especially for modelling and auralisation of 3D spaces. The past few years have seen a rise of interest in wave-based methods, partly driven by the steady increase of commonly available processing power. These methods include finite difference time-domain (FDTD) methods, digital waveguide mesh (DWM) modelling, the finite element method (FEM), the boundary element method (BEM), and the functional transform method (FTM).

This paper focuses on FDTD modelling, which is a good choice for virtual acoustic applications for the following reasons. Firstly, a wide body of knowledge and methods has been developed since the 1960s in the field of electrodynamics, the underlying equations of which are identical to those of acoustic systems. Secondly, unlike finite element methods, FDTD methods tend to use uniform grids, which are more suited to auralisation of virtual spaces with moving sources and receivers. Finally, the formulation and implementation of FDTD models is relatively straight-forward in comparison to some of the other approaches.

Over the past decade, many studies in the area of FDTD and DWM modelling of 2D and 3D acoustic systems have focused on reducing and/or compensating for the dispersion error (see, for example [3, 4]). Recently, more attention has been given to the problem of formulating better numerical approximations of boundaries, and in particular the realization of frequency-dependent boundaries. Generally, realistic boundaries are frequency-dependent even at narrow frequency ranges [5], hence developing accurate formulations 
is an essential ingredient in creating realistic and predictive FDTD simulations.

Strictly speaking, complete physical models of boundaries should include the transmission of waves in the wall. However, simulation results in previous studies [5] have suggested that in many practical cases there is no significant difference if wave propagation in the wall is neglected. In this paper we therefore assume any room surfaces to be locally reacting, i.e. the reflective properties of any point on the wall are completely characterized by an impedance.

With regard to FDTD modelling of a locally reacting surface (LRS), Botteldooren introduced a method for simulating boundaries that can have mass-like and spring-like properties [5]. The main disadvantage of this formulation is that its functionality is constrained by a stability limit that depends on the physical parameters associated with the boundary. Note that Botteldooren's boundary is formulated for a staggered grid, whereas the formulations proposed in this paper are developed for a nonstaggered FDTD grid.

With regard to DWM models, which mathematically can be considered as FDTD models [6, 7], all boundary formulations of the locally reacting surface type that are available in the literature are based on a 1D formulation ${ }^{1}$. That is, wave propagation is locally assumed to be one-dimensional at a boundary, hence the model does not implement wave propagation along boundaries. This simplification is somewhat unphysical, but for high impedances and low angles it still roughly approximates LRS theory. The first boundary of this kind was introduced by Savioja et al. in [9] for the original digital waveguide mesh that is implemented as an FDTD model, thus using Kirchhoff variables, in which case it is often referred to as K-DWM model. In order to obtain frequency-dependent absorption in the wave variables implementation of the digital waveguide mesh (W-DWM), 1D boundary nodes are replaced with digital reflectance filters [10]. The problem of interfacing W-DWM and K-DWM implementations was addressed for the 1D case in [7] with the use of so called KW-pipes. Such 1D KW-converters have later been applied to room acoustics in [11] and [12] in order to connect K-DWM/FDTD room interior implementation with boundary reflectance filters.

In this paper we show that the 1D formulation leads to significant errors in phase and amplitude for low impedances or high angles of incidence, and propose improved numerical formulations of locally reacting surfaces, that can be applied to both FDTD and DWM modelling of acoustic spaces. Preliminary results regarding the comparison of 1D and 2D frequency-independent LRS model have been presented in [13]. The approach taken avoids the unphysicality of the 1D formulation, by combining numerical

\footnotetext{
${ }^{1}$ Spatially integrating methods have been applied in order to model absorbing boundaries in [8]. In that study however, the multi-dimensional wave equation is not preserved at the boundary, and the method proposed in [8] is not suitable for modelling reflective boundaries.
}

versions of the LRS condition and the multi-dimensional (i.e. $2 \mathrm{D}$ or $3 \mathrm{D}$ ) wave equation. Besides using a nonstaggered grid, the formulation also differs from Botteldooren's in that it does not introduce a new stability bound, i.e. the simulation is stable for any physically feasible boundary parameters. The formulations supplied in this paper consider both 2D and 3D systems, including the treatment of corners and boundary edges. In addition, a numerical boundary analysis method is proposed that provides a useful tool for analysis of the numerical reflectance.

The paper is structured as follows. Sections 2 and 3 briefly review the finite difference time domain method and the theory of locally reacting surfaces, respectively. The next two sections discuss the 2D formulation of frequencyindependent and frequency-dependent boundaries, followed by Section 6 that summarizes all 3D formulations. The numerical boundary analysis is proposed in Section 7. The discussion of all results is presented in Section 8, including comparisons between different approaches as well as between experiment and theory. Finally some concluding remarks are presented in Section 9.

\section{The FDTD method}

Sound wave propagation in air is governed by two laws, namely the conservation of mass and the conservation of momentum [14]. The former is expressed by

$$
\nabla p+\rho \frac{\partial \mathbf{u}}{\partial t}=0
$$

and the latter is given by

$$
\frac{\partial p}{\partial t}+\kappa \nabla \mathbf{u}=0
$$

where $p$ denotes the acoustic pressure, $\mathbf{u}$ is the vector particle velocity, $\rho$ is the air density, $\kappa=\rho c^{2}$, and $c$ is the sound velocity. The wave equation can be derived by eliminating the particle velocity from Equation (1) using Equation (2), which yields

$$
\frac{\partial^{2} p}{\partial t^{2}}=c^{2} \nabla^{2} p
$$

Numerical solving the wave equation with the use of the FDTD method relies on approximating time and space derivatives with finite difference (FD) operators. This technique is usually characterized by a regular spatial grid, the size of which depends upon the sampling frequency. The nonstaggered rectilinear standard leapfrog finite difference formulation of the wave equation is obtained by applying centered difference operators to approximate the derivatives in Equation (3), the example second-order accurate approximations applied to discretise the $1 \mathrm{D}$ wave equation are

$$
\frac{\partial^{2} p}{\partial x^{2}}=\frac{p_{l+1}^{n}-2 p_{l}^{n}+p_{l-1}^{n}}{X^{2}}+O\left(X^{2}\right),
$$




$$
\frac{\partial^{2} p}{\partial t^{2}}=\frac{p_{l}^{n+1}-2 p_{l}^{n}+p_{l}^{n-1}}{T^{2}}+O\left(T^{2}\right),
$$

where $X$ denotes the grid spacing and $T$ is the time step. Assuming equal distances between grid points in all directions, the 1D, 2D, and 3D discretised wave equations take the form of Equations (6), (7), and (8), respectively [15]

$$
\begin{aligned}
p_{l}^{n+1}= & \lambda^{2}\left(p_{l+1}^{n}+p_{l-1}^{n}\right)+2\left(1-\lambda^{2}\right) p_{l}^{n}-p_{l}^{n-1}, \\
p_{l, m}^{n+1}= & \lambda^{2}\left(p_{l+1, m}^{n}+p_{l-1, m}^{n}+p_{l, m+1}^{n}+p_{l, m-1}^{n}\right) \\
+ & 2\left(1-2 \lambda^{2}\right) p_{l, m}^{n}-p_{l, m}^{n-1}, \\
p_{l, m, i}^{n+1}= & \lambda^{2}\left(p_{l+1, m, i}^{n}+p_{l-1, m, i}^{n}+p_{l, m+1, i}^{n}\right. \\
& \left.+p_{l, m-1, i}^{n}+p_{l, m, i+1}^{n}+p_{l, m, i-1}^{n}\right) \\
& +2\left(1-3 \lambda^{2}\right) p_{l, m, i}^{n}-p_{l, m, i}^{n-1},
\end{aligned}
$$

where $\lambda=\frac{c T}{X}$ denotes the Courant number, $p_{l, m, i}^{n}$ is the pressure update variable, $n$ is a time index, and $l, m$, and $i$ denote spatial indexes in $x$-, $y$-, and $z$-direction, respectively. The Courant stability condition amounts to $\lambda \leq 1$, $\lambda \leq 1 / \sqrt{2}$ and $\lambda \leq 1 / \sqrt{3}$ for the $1 \mathrm{D}, 2 \mathrm{D}$, and 3D scheme, respectively [15].

An unwanted side effect of using FDTD schemes is the numerical dispersion error, i.e. the numerical phase velocity differs from the theoretical phase velocity, and for 2D and $3 \mathrm{D}$ rooms this phenomenon is generally dependent on frequency and propagation direction [15]. In order to minimize the dispersion error, the Courant number is usually chosen at the stability bound, i.e. $\lambda=1, \lambda=1 / \sqrt{2}$, and $\lambda=1 / \sqrt{3}$ for modelling sound wave propagation in $1 \mathrm{D}, 2 \mathrm{D}$, and $3 \mathrm{D}$ rooms, respectively. For the 1D case, the numerical dispersion actually vanishes for $\lambda=1$. The dispersion equations for the $2 \mathrm{D}$ and $3 \mathrm{D}$ leapfrog rectilinear schemes are respectively given by

$$
\frac{1}{\lambda^{2}} \sin ^{2}\left(\frac{\omega T}{2}\right)=\left[\sin ^{2}\left(\frac{X \widehat{k} \cos \theta}{2}\right)+\sin ^{2}\left(\frac{X \widehat{k} \sin \theta}{2}\right)\right]
$$

$$
\begin{aligned}
\frac{1}{\lambda^{2}} \sin ^{2}\left(\frac{\omega T}{2}\right)= & {\left[\sin ^{2}\left(\frac{X \widehat{k} \cos \theta \cos \phi}{2}\right)\right.} \\
& +\sin ^{2}\left(\frac{X \widehat{k} \sin \theta \cos \phi}{2}\right) \\
& \left.+\sin ^{2}\left(\frac{X \widehat{k} \sin \phi}{2}\right)\right]
\end{aligned}
$$

where $\widehat{k}$ is the discrete-domain wavenumber, and $\theta$ and $\phi$ are the azimuth and elevation angles, respectively [16]. For 2D/3D rooms, more accurate FD schemes based on the rectilinear mesh topology may be applied to reduce the dispersion error; one good example is the compact implicit FD technique which achieves the highest order of accuracy on the smallest mesh system [4].
For top values of the Courant number, the standard leapfrog rectilinear scheme is mathematically equivalent to the rectangular digital waveguide mesh $[6,7]$. Other DWM formulae based on the rectilinear structure such as the interpolated digital waveguide mesh (IDWM) introduced in [3] can also be considered as FDTD methods. Hence the boundary models presented in this paper for the FDTD technique are also directly applicable to the DWM simulations.

FDTD boundary formulations can generally be derived by combining the discretised wave equation with a discretised boundary condition, and realized by updating the boundary nodes (and where required, the associated ghost points) with the resulting boundary update equation. In this paper, all boundary update equations are explicit difference equations. Provided that an unstaggered grid is used, these can in principle be used to compute boundary nodes in all types of FDTD and DWM simulations, including cases where the room interior nodes are computed with an implicit scheme. When alternating direction implicit (ADI) methods are applied, such as in [4], the use of explicit boundary formulations is not merely a choice but a necessity, as the ADI method requires at each time step that the boundary node values are known before solving for the interior nodes.

\section{Locally Reacting Surfaces}

Throughout this paper, reflecting boundary surfaces are assumed to be plane, even though negligible roughness of the surface is permitted as long as its dimensions are much smaller than the shortest wavelength [14]. A reflective boundary can be modelled as a locally reacting surface (LRS), where the normal component of the particle velocity at the surface of the wall depends on the sound pressure in front of the wall element and not on pressure in front of neighbouring elements [14]. This assumption holds for boundaries that are unable to propagate vibrations in the direction parallel to the boundary surface. If we consider a sound wave travelling in a positive $x$-direction, the boundary impedance $Z_{w}$ relates the sound pressure to the flow normal to the wall by

$$
p=Z_{w} u_{x}
$$

where $p$ denotes pressure and $u_{x}$ is the velocity component that is normal to the surface of the boundary. For waves travelling in negative $x$-direction, this changes to $p=-Z_{w} u_{x}$. While the wave equation is derived from the principles of the conservation of mass and the conservation of momentum, only the latter one may be applied in isolation at the boundary [14]. For a boundary normal to the $x$-direction the conservation of momentum equation is

$$
\frac{\partial p}{\partial x}=-\rho \frac{\partial u_{x}}{\partial t} .
$$


Differentiating both sides of Equation (11) yields

$$
\frac{\partial p}{\partial t}=Z_{w} \frac{\partial u_{x}}{\partial t}
$$

Next, subsituting for $\frac{\partial u_{x}}{\partial t}$ in Equation (12) with Equation (13) yields the boundary condition for the right boundary in terms of pressure only

$$
\frac{\partial p}{\partial t}=-c \xi_{w} \frac{\partial p}{\partial x}
$$

where $\xi_{w}=Z_{w} / \rho c$ is the normalised wall impedance, also known as the 'specific acoustic impedance', which completely characterizes the boundary. For the left boundary, the flow is orientated in the opposite direction and hence the minus in Equation (14) should be omitted. The corresponding planar wave reflection coefficient, herein referred to as the reflectance, is related to the specific acoustic impedance and the angle of incidence by [14]

$$
R(\theta)=\frac{\xi_{w} \cos \theta-1}{\xi_{w} \cos \theta+1} .
$$

Equation (15) implies that for a rigrid wall, i.e. very high values of the specific acoustic impedance, we have $R(\theta)=$ 1 for all angles. At the other extreme, the specific acoustic impedance equals the characteristic impedance of air $\left(\xi_{w}=1\right)$, and the boundary is completely absorbent at normal incidence, but not at any other angle. This shows that the locally reacting surface model is a priori not a good basis for deriving an 'anechoic boundary condition'. In summary, the LRS theory provides a simple basis for modelling specular wall reflections, but is less suited to modelling more complex boundaries such as anechoic terminations.

\section{Frequency-independent bound- aries}

Even though real acoustic boundaries are characterized by complex impedances, let us for now consider the simpler case of frequency-independent boundaries in a 2D acoustic space. From a physical point of view, it is logical to treat the discretisation of a locally reacting surface model as a $2 \mathrm{D}$ problem. However, the literature also provides a 1D approach, which is why both cases are discussed here.

\subsection{D formulation}

An FDTD boundary model of a locally reacting surface can be obtained by approximating the first-order spatial and time derivatives in Equation (14) with centered finite difference operators, i.e.

$$
\frac{\partial p}{\partial x}=\frac{p_{l+1, m}^{n}-p_{l-1, m}^{n}}{2 X}+O\left(X^{2}\right)
$$

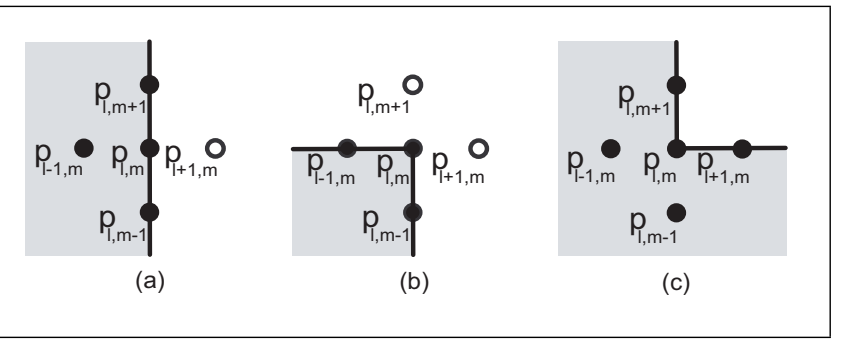

Figure 1: The leapfrog stencil in a 2D rectilinear mesh at: (a) a boundary, (b) an outer corner, (c) an inner corner. Ghost-point nodes are indicated with white-coloured circles and the room interior is indicated by grey shading.

$$
\frac{\partial p}{\partial t}=\frac{p_{l, m}^{n+1}-p_{l, m}^{n-1}}{2 T}+O\left(T^{2}\right)
$$

The resulting equation can be written as an expression for the point lying outside of the modelled space, also referred to as a 'ghost point' [17], which for the right boundary [see Figure 1(a)] yields

$$
p_{l+1, m}^{n}=p_{l-1, m}^{n}+\frac{1}{\lambda \xi_{w}}\left(p_{l, m}^{n-1}-p_{l, m}^{n+1}\right) .
$$

Eliminating the ghost point in the 2D discretised wave equation (7) using Equation (18) yields the following 2D boundary update equation for a right boundary

$$
\begin{aligned}
p_{l, m}^{n+1} & =\left[2\left(1-2 \lambda^{2}\right) p_{l, m}^{n}+\lambda^{2}\left(p_{l, m+1}^{n}+p_{l, m-1}^{n}\right)\right. \\
& \left.+2 \lambda^{2} p_{l-1, m}^{n}+\left(\frac{\lambda}{\xi_{w}}-1\right) p_{l, m}^{n-1}\right] /\left(1+\frac{\lambda}{\xi_{w}}\right) .
\end{aligned}
$$

For both the boundary update equation and the general scheme for updating the room-interior nodes, the upper stability bound is matched by setting $\lambda=\frac{1}{\sqrt{2}}$, which reduces Equation (19) to

$$
p_{l, m}^{n+1}=r\left[2 p_{l-1, m}^{n}+p_{l, m+1}^{n}+p_{l, m-1}^{n}\right]+(1-4 r) p_{l, m}^{n-1},
$$

where $r=\frac{\xi_{w}}{\sqrt{2}+2 \xi_{w}}$. Note that Equation (20) can be efficiently computed in fixed-point implementations using only one multiplier and two bit shift operations. Similar efficient forms can also be found for all boundary update formulae presented in subsequent sections, but will not be explicitly provided as such.

\subsection{D formulation}

For the purpose of comparing the $2 \mathrm{D}$ formulation given above to the $1 \mathrm{D}$ model commonly applied in the digital waveguide mesh approach, we also derive the boundary update equation that follows from making the (unphysical) assumption that waves at the boundary locally travel in $x$ direction only. In that case the discretised 1D wave equation 
(6) applies instead of the 2D wave equation. The starting point for derivation of a 1D boundary model of a locally reacting surface is the same as for the $2 \mathrm{D}$ case, i.e. the discretisation of the boundary condition, which gives Equation (18). However, this time the ghost point is eliminated in the $1 \mathrm{D}$ discretised wave equation (6), which yields

$$
\begin{aligned}
p_{l, m}^{n+1}= & {\left[2 \lambda^{2} p_{l-1, m}^{n}+2\left(1-\lambda^{2}\right) p_{l, m}^{n}\right.} \\
& \left.+\left(\frac{\lambda}{\xi_{w}}-1\right) p_{l, m}^{n-1}\right] /\left(1+\frac{\lambda}{\xi_{w}}\right) .
\end{aligned}
$$

For notational consistence, we keep $m$-indexes at the variables. As discussed in Section 2, it is natural to choose $\lambda=\frac{1}{\sqrt{2}}$ since that value of the Courant number is the upper stability bound for the $2 \mathrm{D}$ rectilinear FDTD scheme given by Equation (7). Alternatively, one could consider that the $1 \mathrm{D}$ wave equation has a stability bound at $\lambda=1$, and locally define the Courant number as such. If we then use Equation (21) and substitute $\xi_{w}=\frac{1+R\left(0^{\circ}\right)}{1-R\left(0^{\circ}\right)}$, where $R\left(0^{\circ}\right)$ is the normal-incidence wall reflectance, the following boundary update formula results

$$
p_{l, m}^{n+1}=\left[1+R\left(0^{\circ}\right)\right] p_{l-1, m}^{n}-R\left(0^{o}\right) p_{l, m}^{n-1} .
$$

This boundary update formula is identical to the formula used in many previous studies (see for example [18, 19]), and was originally derived from a digital waveguide mesh perspective in [9]. For a given sample rate and a constant sound speed, $\lambda$ defines the distances between grid points. Therefore, choosing $\lambda=1$ at the boundary implies that the distance from the boundary node to the nearest neighbouring node in the medium is smaller than distances between nodes in the medium. As depicted in Figure 2(c), when updating an interior node that is located next to the boundary, the distance between this node and the boundary node is larger than the distance in the opposite direction, i.e. from the boundary node to the same interior node when updating the boundary node. On the other hand, by setting $\lambda=\frac{1}{\sqrt{2}}$ the grid spacing is uniform, but this choice should result in an increase in numerical error at the boundary as this value is much below the upper stability bound for the 1D wave equation. As will be shown in Section 8, the 1D formulation leads to erroneous results for either choice of the Courant number, which is fundamentally due to the unphysical assumption of $1 \mathrm{D}$ wave propagation at the boundary.

\subsection{Corners}

In the $1 \mathrm{D}$ formulation, the boundary itself is effectively not regarded as part of the medium. Hence at outer corners [see Figure 1(b)] the wave equation applies in neither $x$ - nor $y$ direction. Therefore outer corner nodes are not updated (i.e. kept at zero) when using a 1D boundary formulation, as depicted in Figure 2(b). Simple experiments have shown

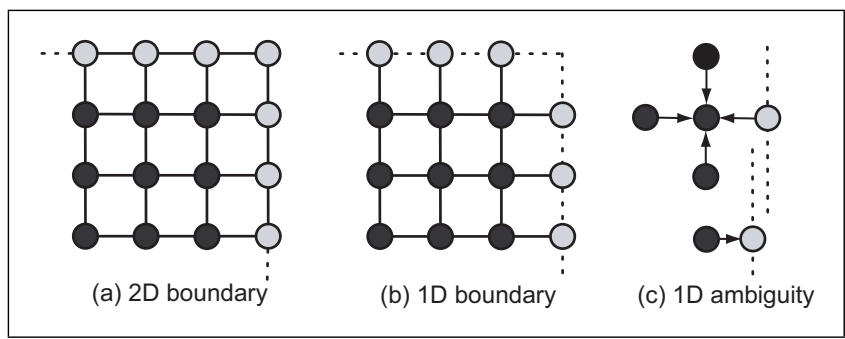

Figure 2: $2 \mathrm{D}$ and $1 \mathrm{D}$ terminations of a 2D mesh structure. Boundary nodes are indicated with grey-coloured circles and black arrows point to the updated node in (c).

that this leads to spurious results when a wave hits a corner; for example when a plane wave travelling in $x$-direction reflects from a corner, some wave energy is directed in the $y$-direction.

No such problems arise with the $2 \mathrm{D}$ formulation. The derivation of the update equation for a top-right outer corner now starts from the principle that two boundary conditions have to be satisfied simultaneously at the boundary node, namely

$$
\frac{\partial p}{\partial t}=-c \xi_{x} \frac{\partial p}{\partial x}, \quad \frac{\partial p}{\partial t}=-c \xi_{y} \frac{\partial p}{\partial y}
$$

where $\xi_{x}$ denotes the normalised impedance in $x$-direction and $\xi_{y}$ is the normalised impedance in $y$-direction, respectively. Next, substituting for both ghost points in the 2D discrete wave equation (7) with discrete versions of Equations (23), yields

$$
\begin{aligned}
p_{l, m}^{n+1} & =\left[2 \lambda^{2}\left(p_{l-1, m}^{n}+p_{l, m-1}^{n}\right)+2\left(1-2 \lambda^{2}\right) p_{l, m}^{n}\right. \\
& \left.+\left(\frac{\lambda}{\xi_{x}}+\frac{\lambda}{\xi_{y}}-1\right) p_{l, m}^{n-1}\right] /\left(1+\frac{\lambda}{\xi_{x}}+\frac{\lambda}{\xi_{y}}\right) .
\end{aligned}
$$

Hence the corner node can now be updated, as shown in Figure 1(b). Other outer corners, which for 2D include boundaries left, above, and below the room interior are derived in the same manner, where differences in the final formulae only occur in the form of changes in sign and index; all coefficients remain the same.

At inner corners, also referred to as re-entrant corners, neither of the two boundary conditions apply. There are also no ghost points to eliminate, as shown in Figure 1(c). Hence an inner corner node can be updated with the discretised 2D wave equation (7).

\section{Frequency-dependent boundaries}

In general, real acoustic boundaries are frequencydependent, which means that the reflected wave has both phase and amplitude that differ from those of an incident 
wave, and such changes vary with frequency [14]. The 2D LRS model presented in Section 4 can be extended by replacing the real-valued wall impedance with a frequencydependent, complex-valued impedance. As a first approximation, one can distinguish two basic types of such boundaries, namely boundaries that behave spring-like and those that behave mass-like [5]. The former can be used to model a thin absorbing layers stretched on the much harder boundaries; seat, floor and wall coverings are good examples. The latter boundary type models heavy porous layers such as curtains or light nonstiff walls. More complex boundaries can be treated as a superposition of these two types [5].

As will be shown in Section 8, the 1D boundary formulation of frequency-independent boundaries suffers from severe amplitude and phase errors. For this reason the 1D approach is not considered for frequency-dependent boundaries.

\subsection{Boundary formulation}

For a spring-like boundary, the impedance is defined as $Z_{w}(s)=R+K / s$, where $R$ denotes resistance, $K$ is the spring constant, and $s$ is the Laplace frequency variable. Mass-like boundaries are defined by $Z_{w}(s)=R+M s$, where $M$ denotes the mass per unit area. For some realistic acoustic boundaries, such as may be the case for parquet, both behaviours need to be modelled. Therefore a fairly general boundary model can be defined by the impedance

$$
Z_{w}(s)=R+M s+\frac{K}{s}
$$

Inserting this impedance into Equation (14) yields the boundary condition for a right boundary

$$
\frac{\partial p}{\partial t}=-c\left(\frac{R}{\rho c} \frac{\partial p}{\partial x}+\frac{M}{\rho c} \frac{\partial p^{2}}{\partial x \partial t}+\frac{K}{\rho c} \int_{-\infty}^{t} \frac{\partial p}{\partial x} d t\right),
$$

Centered finite difference operators [Equations (16) and (17)] are applied to approximate the first-order derivatives $\frac{\partial p}{\partial t}$ and $\frac{\partial p}{\partial x}$ in Equation (26). Concerning a numerical integration method, we propose the use of a composite trapezoidal rule with subintervals equal to time steps. Trapezoidal integration is mathematically equivalent to the bilinear transform $s=\frac{2}{T} \frac{1-z^{-1}}{1+z^{-1}}$ applied to $y=x / s$, which yields

$$
y^{n}=T \frac{x^{n}+x^{n-1}}{2}+y^{n-1},
$$

and therefore the numerical integration can be written

$$
\frac{K}{\rho c} \int_{-\infty}^{t} p_{l, m}^{n} d t=\frac{T K}{2 \rho c} \sum_{i=-\infty}^{n}\left(p_{l, m}^{i}+p_{l, m}^{i-1}\right) .
$$

Similarly to the integration method, the bilinear transform is applied to approximate the time differentiation in the mixed term $\frac{\partial^{2} p}{\partial x \partial t}$. This can be mathematically formulated as an application of the bilinear transform to $y=x s$, which results in

$$
y^{n}=\frac{2}{T}\left(x^{n}-x^{n-1}\right)-y^{n-1} .
$$

This 'digitisation' of the function of the spring- and massterms can be seen as an acoustic analogy to wave digital filter theory, in which the bilinear transform is used to map electrically analogous circuit elements such as inductors and capacitors into their wave digital equivalents [15]. As such, the proposed method for integration and differentiation has some of the associated advantages, which include the property of mapping stable analogue systems to stable digital systems. Another important feature of the proposed discretisation is that an implicit formulation is avoided. Applying these transformations to Equation (26) yields

$p_{l, m}^{n+1}-p_{l, m}^{n-1}=\lambda\left[a_{R}\left(p_{l-1, m}^{n}-p_{l+1, m}^{n}\right)+a_{K} S_{K}^{n}-a_{M} S_{M}^{n}\right]$,

where the parameters $a_{R}, a_{K}$, and $a_{M}$ are given in Table 1. The new variable $S_{K}$ is introduced to store the result of summation 'up to now', which is given by

$$
S_{K}^{n}=p_{l-1, m}^{n}-p_{l+1, m}^{n}+p_{l-1, m}^{n-1}-p_{l+1, m}^{n-1}+S_{K}^{n-1},
$$

and the new variable $S_{M}$ is introduced for storage of the results of the formula

$$
S_{M}^{n}=p_{l+1, m}^{n}-p_{l-1, m}^{n}+p_{l-1, m}^{n-1}-p_{l+1, m}^{n-1}-S_{M}^{n-1} .
$$

In order to obtain the formula for the ghost point $p_{l+1, m}$ at time step $n$, the new storage variables $S_{K}$ and $S_{M}$ are next written explicitly in Equation (30), which after some basic algebra results in the ghost point formula

$$
\begin{aligned}
p_{l+1, m}^{n}= & p_{l-1, m}^{n}+\frac{1}{\lambda a}\left(p_{l, m}^{n-1}-p_{l, m}^{n+1}\right)+\frac{a_{K}-a_{M}}{a} \\
& \cdot\left(p_{l-1, m}^{n-1}-p_{l+1, m}^{n-1}\right)+\frac{a_{K}}{a} S_{K}^{n-1}+\frac{a_{M}}{a} S_{M}^{n-1},
\end{aligned}
$$

Note that the boundary condition is still centered at time step $n$ resulting in a second-order accurate approximation, and the grouping of terms in Equation (33) results in a structurally stable formulation. The update formula for the right boundary node is then obtained by substituting for the ghost point $p_{l+1, m}$ in the discrete-domain 2D wave equation (7) with the boundary condition (33), which yields

$$
\begin{aligned}
p_{l, m}^{n+1} & =\left[\lambda^{2}\left(2 p_{l-1, m}^{n}+p_{l, m+1}^{n}+p_{l, m-1}^{n}\right)+2\left(1-2 \lambda^{2}\right) p_{l, m}^{n}\right. \\
& +\left(\frac{\lambda}{a}-1\right) p_{l, m}^{n-1}+\frac{\lambda^{2}\left(a_{K}-a_{M}\right)}{a}\left(p_{l-1, m}^{n-1}-p_{l+1, m}^{n-1}\right) \\
& \left.+\frac{\lambda^{2} a_{K}}{a} S_{K}^{n-1}+\frac{\lambda^{2} a_{M}}{a} S_{M}^{n-1}\right] /\left(1+\frac{\lambda}{a}\right) .
\end{aligned}
$$

The boundary formulation requires the update of the boundary node, the ghost point, and the new variables $S_{K}$ and $S_{M}$ 
Table 1: Numerical boundary parameters for a complex impedance.

\begin{tabular}{|c|c|}
\hline Parameter & Description \\
\hline \hline$a_{R}=\frac{R}{\rho c}$ & Specific resistance \\
\hline$a_{K}=\frac{T K}{2 \rho c}$ & Specific spring constant times $\mathrm{T} / 2$ \\
\hline$a_{M}=\frac{2 M}{T \rho c}$ & Two times specific mass divided by $\mathrm{T}$ \\
\hline$a=a_{R}+a_{K}+a_{M}$ & Sum of the previous parameters \\
\hline
\end{tabular}

(in that order) at each time step according to the aforementioned equations. This way the computed values $S_{K}$ and $S_{M}$ are only applied in the next time step. Note that only one previous value needs to be stored for the ghost point.

\subsection{Corners}

The $2 \mathrm{D}$ formulation of corners is accomplished similarly to the frequency-independent case. That is, an inner corner is updated with the 2D wave equation (7). An outer corner has two boundary conditions (in $x$ - and $y$-direction), the discretised versions of which are used for the elimination of the two ghost points in the discretised $2 \mathrm{D}$ wave equation. The resulting update formula for the top-right outer corner node is

$$
\begin{aligned}
p_{l, m}^{n+1} & =\left[\lambda^{2}\left(2 p_{l-1, m}^{n}+2 p_{l, m-1}^{n}+g_{x}^{n-1}+g_{y}^{n-1}\right)\right. \\
& +\left(\frac{\lambda}{a_{x}}+\frac{\lambda}{a_{y}}-1\right) p_{l, m}^{n-1} \\
& \left.+2\left(1-2 \lambda^{2}\right) p_{l, m}^{n}\right] /\left(\frac{\lambda}{a_{x}}+\frac{\lambda}{a_{y}}+1\right),
\end{aligned}
$$

where the ghost points are computed as

$$
\begin{aligned}
& p_{l+1, m}^{n}=p_{l-1, m}^{n}+\frac{1}{\lambda a_{x}}\left(p_{l, m}^{n-1}-p_{l, m}^{n+1}\right)+g_{x}^{n-1}, \\
& p_{l, m+1}^{n}=p_{l, m-1}^{n}+\frac{1}{\lambda a_{y}}\left(p_{l, m}^{n-1}-p_{l, m}^{n+1}\right)+g_{y}^{n-1},
\end{aligned}
$$

with

$$
\begin{aligned}
g_{x}^{n-1}= & \frac{a_{K x}-a_{M x}}{a}\left(p_{l-1, m}^{n-1}-p_{l+1, m}^{n-1}\right) \\
& +\frac{a_{K x}}{a} S_{K x}^{n-1}+\frac{a_{M x}}{a} S_{M x}^{n-1}, \\
g_{y}^{n-1}= & \frac{a_{K y}-a_{M y}}{a}\left(p_{l, m-1}^{n-1}-p_{l, m+1}^{n-1}\right) \\
& +\frac{a_{K y}}{a} S_{K y}^{n-1}+\frac{a_{M y}}{a} S_{M y}^{n-1},
\end{aligned}
$$

where the four variables $S_{K x}, S_{M x}, S_{K y}$, and $S_{M y}$ are computed recursively, in the same way as Equations (31) and (32).

\section{Boundaries in 3D}

\subsection{Boundary formulation}

In the $3 \mathrm{D}$ formulation, the discretised boundary condition given by Equation (26) is combined with the 3D discretised wave equation (8). From there on, the derivation is analogous to the derivation of the $2 \mathrm{D}$ model in Section 5.1. In this case, the ghost point formula reads

$$
\begin{aligned}
p_{l+1, m, i}^{n}= & p_{l-1, m, i}^{n}+\frac{1}{\lambda a}\left(p_{l, m, i}^{n-1}-p_{l, m, i}^{n+1}\right)+\frac{a_{K}-a_{M}}{a} \\
& \cdot\left(p_{l-1, m, i}^{n-1}-p_{l+1, m, i}^{n-1}\right)+\frac{a_{K}}{a} S_{K}^{n-1}+\frac{a_{M}}{a} S_{M}^{n-1},
\end{aligned}
$$

and the variables $S_{K}$ and $S_{M}$ are defined as

$$
\begin{aligned}
& S_{K}^{n}=p_{l-1, m, i}^{n}-p_{l+1, m, i}^{n}+p_{l-1, m, i}^{n-1}-p_{l+1, m, i}^{n-1}+S_{K}^{n-1}, \\
& S_{M}^{n}=p_{l+1, m, i}^{n}-p_{l-1, m, i}^{n}+p_{l-1, m, i}^{n-1}-p_{l+1, m, i}^{n-1}-S_{M}^{n-1} .
\end{aligned}
$$

Finally, elimination of the ghost point in the 3D discrete wave equation (8) using the boundary condition given by Equation (40) yields the 3D boundary update formula

$$
\begin{aligned}
p_{l, m, i}^{n+1} & =\left[\lambda ^ { 2 } \left(2 p_{l-1, m, i}^{n}+p_{l, m+1, i}^{n}+p_{l, m-1, i}^{n}+p_{l, m, i+1}^{n}\right.\right. \\
& \left.+p_{l, m, i-1}^{n}\right)+2\left(1-3 \lambda^{2}\right) p_{l, m, i}^{n}+\left(\frac{\lambda}{a}-1\right) p_{l, m, i}^{n-1} \\
& +\frac{\lambda^{2}\left(a_{K}-a_{M}\right)}{a}\left(p_{l-1, m, i}^{n-1}-p_{l+1, m, i}^{n-1}\right) \\
& \left.+\frac{\lambda^{2} a_{K}}{a} S_{K}^{n-1}+\frac{\lambda^{2} a_{M}}{a} S_{M}^{n-1}\right] /\left(1+\frac{\lambda}{a}\right)
\end{aligned}
$$

This 3D formulation requires computing the formulae for the boundary node, the ghost point, variables $S_{K}$ and $S_{M}$ at each time step with Equations (43), (40), (41), and (42), respectively. The frequency-independent version of the $3 \mathrm{D}$ boundary model is obtained by simply setting $a_{K}=0$ and $a_{M}=0$. In that case only the boundary node given by Equation (43) needs updating.

\subsection{Corners}

The formulation of an outer corner in a 3D system relies on applying a 3D wave equation together with three boundary conditions simultaneously. In the 3D case, three ghost points have to be eliminated in the discrete $3 \mathrm{D}$ wave equation (8) with formulae obtained from each boundary condition separately, namely in $x$-, $y$ - and $z$-direction. This yields the following update formula

$$
\begin{aligned}
p_{l, m, i}^{n+1} & =\left[\lambda ^ { 2 } \left(2 p_{l-1, m, i}^{n}+2 p_{l, m-1, i}^{n}+2 p_{l, m, i-1}^{n}+g_{x}^{n-1}\right.\right. \\
& \left.+g_{y}^{n-1}+g_{z}^{n-1}\right)+\left(\frac{\lambda}{a_{x}}+\frac{\lambda}{a_{y}}+\frac{\lambda}{a_{z}}-1\right) p_{l, m, i}^{n-1} \\
& \left.+2\left(1-3 \lambda^{2}\right) p_{l, m, i}^{n}\right] /\left(1+\frac{\lambda}{a_{x}}+\frac{\lambda}{a_{y}}+\frac{\lambda}{a_{z}}\right) .
\end{aligned}
$$


At an $x-y$ edge of a 3D space, two boundary conditions together with the wave equation are applied, which results in

$$
\begin{aligned}
p_{l, m, i}^{n+1} & =\left[\lambda ^ { 2 } \left(2 p_{l-1, m, i}^{n}+2 p_{l, m-1, i}^{n}+p_{l, m, i-1}^{n}+p_{l, m, i+1}^{n}\right.\right. \\
& \left.+g_{x}^{n-1}+g_{y}^{n-1}\right)+\left(\frac{\lambda}{a_{x}}+\frac{\lambda}{a_{y}}\right) p_{l, m, i}^{n-1} \\
& \left.+2\left(1-3 \lambda^{2}\right) p_{l, m, i}^{n}\right] /\left(1+\frac{\lambda}{a_{x}}+\frac{\lambda}{a_{y}}\right)
\end{aligned}
$$

with the ghost points computed using the formulae

$$
\begin{aligned}
& p_{l+1, m, i}^{n}=p_{l-1, m, i}^{n}+\frac{1}{\lambda a_{x}}\left(p_{l, m, i}^{n-1}-p_{l, m, i}^{n+1}\right)+g_{x}^{n-1}, \\
& p_{l, m+1, i}^{n}=p_{l, m-1, i}^{n}+\frac{1}{\lambda a_{y}}\left(p_{l, m, i}^{n-1}-p_{l, m, i}^{n+1}\right)+g_{y}^{n-1}, \\
& p_{l, m, i+1}^{n}=p_{l, m, i-1}^{n}+\frac{1}{\lambda a_{y}}\left(p_{l, m, i}^{n-1}-p_{l, m, i}^{n+1}\right)+g_{z}^{n-1},
\end{aligned}
$$

where $g_{x}, g_{y}$, and $g_{z}$ are computed in the form of Equations (38) and (39), and the six variables $S_{K x}, S_{M x}, S_{K y}, S_{M y}$, $S_{K z}$, and $S_{M z}$ are computed recursively, in the same way as Equations (31) and (32). As is the case for 2D systems, inner corner nodes are updated using the discretised wave equation (8).

\section{$7 \quad$ Numerical boundary analysis}

The effective reflectance of a numerical boundary (hereafter referred to as the "numerical reflectance") can be predicted in an exact manner, and can also be used to determine whether FDTD simulations using the 2D/3D boundary formulations are stable. To derive such analytical formulae, methods that are very similar to standard methods for the analysis of stability and numerical dispersion in FDTD schemes (see e.g. [16]) or the GKSO (Gustafsson-KreissSundström-Osher) method for boundary condition analysis in the frequency domain [20] can be applied. We will refer to this procedure as "numerical boundary analysis" (NBA).

\subsection{D boundary}

Consider a boundary in an $x-y$ plane that is parallel to the $y$ axis and located at $x=0$. An incident wave of frequency $\omega$ propagating at an angle of incidence $\theta$ towards the boundary from the area $x<0$ can be expressed as [14]

$$
p\left(x^{\prime}, t\right)=p_{0} e^{j \omega t} e^{-j k x^{\prime}},
$$

where $p_{0}$ is the wave amplitude, $k=\omega / c$ denotes the wavenumber, and $x^{\prime}=(x \cos \theta+y \sin \theta)$. For the reflected wave, the sign in $x$-direction is reversed and the pressure amplitude is multiplied by the reflectance. The total sound pressure at the boundary is the sum of the incident and reflected sound pressure [14]

$p(x, y, t)=p_{0} e^{j \omega t} e^{-j k y \sin \theta}\left(e^{-j k x \cos \theta}+R e^{j k x \cos \theta}\right)$.

The equivalent pressure in the discrete domain is written as

$p_{l, m}^{n}=p_{0} e^{j \omega n T} e^{-\widehat{j k m} X \sin \theta}\left(e^{-\widehat{j k l} X \cos \theta}+\widehat{R} e^{\widehat{j k l} X \cos \theta}\right)$

where $\widehat{R}$ is the numerical reflectance and $\widehat{k}$ is the discretedomain wavenumber that has to be computed from Equation (9). On the basis that all pressure variables involved obey the difference equation associated with the leapfrog rectilinear scheme, Equation (51) can also be used for deriving all of the other discrete-domain pressure values in the boundary update equation, for example

$$
\begin{aligned}
& p_{l, m}^{n+1}=e^{j \omega T} p_{l, m}^{n}=z p_{l, m}^{n}, \\
& p_{l, m}^{n-1}=e^{-j \omega T} p_{l, m}^{n}=z^{-1} p_{l, m}^{n}, \\
& p_{l-1, m}^{n}=p_{0} e^{j \omega n T} e^{-\widehat{k k m X} \sin \theta} \\
& \cdot\left(e^{-j \widehat{k}(l-1) X \cos \theta}+\widehat{R} e^{j \widehat{k}(l-1) X \cos \theta}\right) \\
& p_{l+1, m}^{n}=p_{0} e^{j \omega n T} e^{-\hat{k} m X \sin \theta} \\
& \left(e^{-j \widehat{k}(l+1) X \cos \theta}+\widehat{R} e^{\widehat{j k}(l+1) X \cos \theta}\right)
\end{aligned}
$$

Let us first consider the frequency-independent boundary model given by Equation (19). After substitution of all pressure values, the next step is to set $l=0$, which corresponds to $x=0$ at a boundary. Solving then for the numerical reflectance $\widehat{R}$ gives

$$
\begin{aligned}
\widehat{R}(z, \theta) & =-\left\{\left(1+\frac{\lambda}{\xi_{w}}\right) z-\left[2 \lambda^{2} C+\lambda^{2}\left(D+D^{-1}\right)\right.\right. \\
& \left.\left.+2\left(1-2 \lambda^{2}\right)\right]+\left(1-\frac{\lambda}{\xi_{w}}\right) z^{-1}\right\} \\
& /\left\{\left(1+\frac{\lambda}{\xi_{w}}\right) z-\left[2 \lambda^{2} C^{-1}+\lambda^{2}\left(D+D^{-1}\right)\right.\right. \\
& \left.\left.+2\left(1-2 \lambda^{2}\right)\right]+\left(1-\frac{\lambda}{\xi_{w}}\right) z^{-1}\right\}
\end{aligned}
$$

where $C=e^{j \widehat{k} X \cos \theta}$ and $D=e^{j \widehat{k} X \sin \theta}$. Note that the substitution of discrete-domain pressure values in the above procedure is valid only when the $2 \mathrm{D}$ (or 3D) discretised wave equation is imposed on the boundary node, which means that it is connected to adjacent nodes on the boundary. Hence this method is not suitable for the evaluation of a $1 \mathrm{D}$ boundary formulation in a 2D/3D context (this includes all existing DWM boundary formulations).

For the 2D frequency-dependent boundary, the numerical boundary analysis is performed analogous to the frequency- 
independent case. The variable $S_{K}$ can in this case be written as

$$
S_{K}^{n}-S_{K}^{n-1}=p_{l-1, m}^{n}-p_{l+1, m}^{n}+p_{l-1, m}^{n-1}-p_{l+1, m}^{n-1} .
$$

For a plane wave of frequency $\omega$, we can write this as

$$
S_{K}^{n}=\left(p_{l-1, m}^{n}-p_{l+1, m}^{n}\right) \frac{1+z^{-1}}{1-z^{-1}} .
$$

where $z=e^{j \omega T}$. Similarly, the expression for $S_{M}$ is given by

$$
S_{M}^{n}=\left(p_{l+1, m}^{n}-p_{l-1, m}^{n}\right) \frac{1-z^{-1}}{1+z^{-1}},
$$

Inserting these values in Equation (34) and solving for the numerical reflectance $\widehat{R}$ yields the final NBA formula

$$
\begin{aligned}
\widehat{R}(z, \theta)= & -\left\{\left(1+\frac{\lambda}{a}\right) z-\left[2 \lambda^{2} C+\lambda^{2}\left(D+D^{-1}\right)\right.\right. \\
& \left.+2\left(1-2 \lambda^{2}\right)\right]+z^{-1}\left[\left(1-\frac{\lambda}{a}\right)+\left(C^{-1}-C\right)\right. \\
& \left.\left.\cdot\left(\frac{\lambda^{2} a_{K}}{a} \frac{2}{1-z^{-1}}-\frac{\lambda^{2} a_{M}}{a} \frac{2}{1+z^{-1}}\right)\right]\right\} \\
& /\left\{\left(1+\frac{\lambda}{a}\right) z-\left[2 \lambda^{2} C^{-1}+\lambda^{2}\left(D+D^{-1}\right)\right.\right. \\
& \left.+2\left(1-2 \lambda^{2}\right)\right]+z^{-1}\left[\left(1-\frac{\lambda}{a}\right)+\left(C-C^{-1}\right)\right. \\
& \left.\left.\cdot\left(\frac{\lambda^{2} a_{K}}{a} \frac{2}{1-z^{-1}}-\frac{\lambda^{2} a_{M}}{a} \frac{2}{1+z^{-1}}\right)\right]\right\} .(60)
\end{aligned}
$$

Note that Equation (60) follows directly from the numerical boundary analysis applied to Equation (34). However, it can also be rewritten in the mathematically equivalent simple form given by Equation (56), where the specific acoustic impedance is defined as $\xi_{w}(s)=(R+M s+K / s) / \rho c$ and represented in the $z$-domain as

$$
\xi_{w}(z)=\frac{a+2\left(a_{K}-a_{M}\right) z^{-1}+\left(a-2 a_{R}\right) z^{-2}}{1-z^{-2}} .
$$

\subsection{Stability}

Having obtained a formal expression for the numerical reflectance, it can now be shown that the 2D boundary represents a passive termination. Such a stability proof is similar to the standard procedure of proving the stability of FDTD schemes with the use of Von Neumann analysis [16] or the GKSO method for checking the stability of boundary conditions for finite difference schemes [20]. For $\lambda=\frac{1}{\sqrt{2}}$, we may write Equation (56) as

$$
\widehat{R}(z, \theta)=-\frac{Q-C}{Q-C^{-1}},
$$

where for any wavenumber $-\pi / X \leq \widehat{k} \leq \pi / X$, we have

$$
Q=2 \cos (\omega T)-\cos (\widehat{k} X \sin \theta)+j \frac{\sqrt{2}}{\xi_{w}} \sin (\omega T) .
$$

Since the boundary impedance can in a general case be complex-valued, i.e. we may write $\xi_{w}(z)=a_{w}+j b_{w}$. Its multiplicative reciprocal is given as

$$
\frac{1}{\xi_{w}(z)}=\frac{a_{w}}{a_{w}^{2}+b_{w}^{2}}-j \frac{b_{w}}{a_{w}^{2}+b_{w}^{2}},
$$

which now defines the variable $Q$ as

$$
\begin{aligned}
Q & =\left[2 \cos (\omega T)-\cos (\widehat{k} X \sin \theta)+\frac{\sqrt{2} b_{w}}{a_{w}^{2}+b_{w}^{2}} \sin (\omega T)\right] \\
& +j\left[\frac{\sqrt{2} a_{w}}{a_{w}^{2}+b_{w}^{2}} \sin (\omega T)\right] .
\end{aligned}
$$

The boundary is passive if $|\widehat{R}(z, \theta)| \leq 1$, which can also be expressed as

$$
|Q-C| \leq\left|Q-C^{-1}\right|
$$

Taking the square of both the left-hand and the right-hand side of Equation (66) yields

$$
\begin{aligned}
|Q-C|^{2} & =[\operatorname{Re}\{Q\}-\cos (\widehat{k} X \cos \theta)]^{2} \\
& +\left[\frac{\sqrt{2} a_{w}}{a_{w}^{2}+b_{w}^{2}} \sin (\omega T)-\sin (\widehat{k} X \cos \theta)\right]^{2}
\end{aligned}
$$

and

$$
\begin{aligned}
\left|Q-C^{-1}\right|^{2} & =[\operatorname{Re}\{Q\}-\cos (\widehat{k} X \cos \theta)]^{2} \\
& +\left[\frac{\sqrt{2} a_{w}}{a_{w}^{2}+b_{w}^{2}} \sin (\omega T)+\sin (\widehat{k} X \cos \theta)\right]^{2}
\end{aligned}
$$

where $\operatorname{Re}\{Q\}$ denotes the real part of $Q$. Inserting Equations (67) and (68) into (66) and applying some mathematical manipulations, the following condition results

$$
\begin{aligned}
& -\sqrt{2} \frac{a_{w}}{a_{w}^{2}+b_{w}^{2}} \sin (\omega T) \sin (\widehat{k} X \cos \theta) \\
\leq & \sqrt{2} \frac{a_{w}}{a_{w}^{2}+b_{w}^{2}} \sin (\omega T) \sin (\widehat{k} X \cos \theta) .
\end{aligned}
$$

Since $\sin (\omega T) \geq 0$ for frequencies up to Nyquist and $\sin (\widehat{k} X \cos \theta) \geq 0$ is satisfied for all angles $-\pi / 2<\theta \leq$ $\pi / 2$, Equation (69) reduces to

$$
a_{w} \geq 0,
$$

which from the definition of a positive real impedance [21] is always satisfied. Since the medium itself is lossless (none of the FDTD schemes cause numerical attenuation), it follows that for $\lambda=\frac{1}{\sqrt{2}}$ the simulation as a whole is always stable. The passivity proof can also be shown for $\lambda<\frac{1}{\sqrt{2}}$, but is omitted here for brevity. 


\subsection{D boundary}

In a rectangular $3 \mathrm{D}$ coordinate system $(x, y, z)$ with a wall normal to this system and located at $x=0$, an incident wave propagating in a positive $x$-direction is given by Equation (49) with $x^{\prime}=(x \cos \theta \cos \phi+y \sin \theta \cos \phi+z \sin \phi)$, where $\theta$ and $\phi$ are the azimuth and elevation angles, respectively. The total sound pressure in the standing wave in the plane of the boundary is given as

$$
\begin{aligned}
p & =p_{0} e^{j \omega t} e^{-j k y \sin \theta \cos \phi} e^{-j k z \sin \phi} \\
& \cdot\left(e^{-j k x \cos \theta \cos \phi}+R e^{j k x \cos \theta \cos \phi}\right) .
\end{aligned}
$$

If we then discretise Equation (71) and write out the formulae in a similar manner to the derivation of expressions in Section 7.1, the NBA formula for Equation (43) results

$$
\begin{aligned}
\widehat{R}(z, \theta, \phi)= & -\left\{\left(1+\frac{\lambda}{a}\right) z-\left[2 \lambda^{2} C+\lambda^{2}\left(D+D^{-1}\right)\right.\right. \\
& \left.+\lambda^{2}\left(E+E^{-1}\right)+2\left(1-3 \lambda^{2}\right)\right]+z^{-1}\left[\left(1-\frac{\lambda}{a}\right)\right. \\
& +\left(\frac{\lambda^{2} a_{K}}{a} \frac{2}{1-z^{-1}}-\frac{\lambda^{2} a_{M}}{a} \frac{2}{1+z^{-1}}\right. \\
& \left.\left.\left.\cdot\left(C^{-1}-C\right)\right)\right]\right\} /\left\{\left(1+\frac{\lambda}{a}\right) z\right. \\
& -\left[2 \lambda^{2} C^{-1}+\lambda^{2}\left(D+D^{-1}\right)+\lambda^{2}\left(E+E^{-1}\right)\right. \\
& \left.+2\left(1-3 \lambda^{2}\right)\right]+z^{-1}\left[\left(1-\frac{\lambda}{a}\right)+\left(C-C^{-1}\right)\right. \\
& \left.\left.\cdot\left(\frac{\lambda^{2} a_{K}}{a} \frac{2}{1-z^{-1}}-\frac{\lambda^{2} a_{M}}{a} \frac{2}{1+z^{-1}}\right)\right]\right\},(72)
\end{aligned}
$$

where $C=e^{\widehat{j k} X \cos \theta \cos \phi}, D=e^{\widehat{j k} X \sin \theta \cos \phi}$, and $E=$ $e^{j \widehat{k} X \sin \phi}$. In this case the wavenumber $\widehat{k}$ has to be computed from Equation (10). As for the 2D case, the NBA reflectance magnitude can be shown to be less or equal to unity for all wavenumbers and angles of incidence for $\lambda \leq \frac{1}{\sqrt{3}}$.

\section{Results}

\subsection{Numerical Experiments}

In order to investigate the properties of numerical boundary formulations, a 2D simulation procedure for determining the numerical reflectance was designed. In this procedure, simulations are executed using a grid consisting of $1800 \times 1400$ nodes. For most simulations, a fourth-order accurate compact implicit ADI scheme [4] was used, but in specific cases the standard leap-frog scheme was applied; the ADI scheme generally gives better reflectance results due to lower numerical dispersion, which helps in creating more plane wavefronts. Furthermore, the ADI scheme

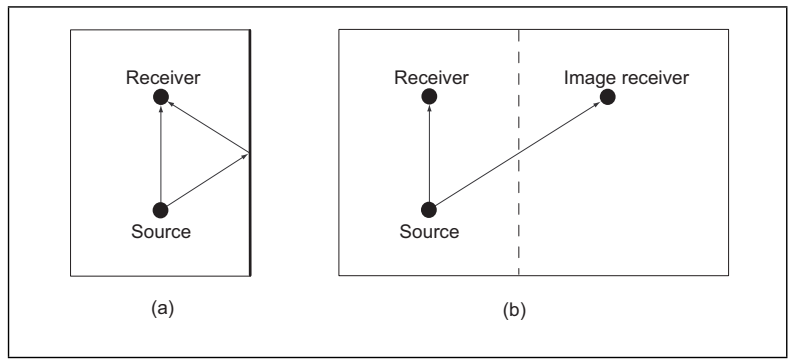

Figure 3: Schematic depiction of the simulation setup used in the numerical experiments.

was applied for comparison of the 2D and 1D boundary models as it requires shorter simulation time needed for the whole wavelet to arrive at a receiver position than the standard leapfrog scheme room interior implementation. The size of the modelled room and the simulation time (2000 samples at the sample rate of $4 \mathrm{kHz}$ ) were selected in such a way that only reflections from the investigated boundary can reach a receiver position. The simulation time was chosen sufficiently long so that the whole wavelet can reach a receiver. The mesh was initialized with a sharp impulse injected into a grid point. The source position was chosen so that 1) a constant distance of 400 grid points from the centre of the investigated wall was preserved, and 2) the incident waves at the following angles of incidence $\theta=$ $0^{\circ}, 15^{\circ}, 30^{\circ}, 45^{\circ}, 60^{\circ}, 75^{\circ}$, resulted. Note that by placing the reflecting boundary far enough from the sound source, the curvature of the wavefront arriving at a boundary may be neglected. The exception was for $\theta=75^{\circ}$, where in order to maintain a flatter wavefront, the size of the modelled space was increased to $2800 \times 2100$, the distance from the source was increased to 700 points, and the simulation time was extended to 3500 samples. When the leapfrog scheme was used for the room interior implementation, the setup was altered in order to deal with the stronger dispersion error (which affects the shape of the wavefront and leads to longer tails in the obtained signals). In that scenario, the domain consisted of $2800 \times 2100$ nodes, the distance from a source to the centre of the boundary was reduced to 100 points, and the simulation time extended to 3700 samples in order to capture the whole wavelet reaching a receiver after over 12 times the wavefront arrival time.

Each reflectance test procedure consists of two simulations, the setup of which is presented in Figure 3. Firstly, the investigated boundary is located in the middle of the room, and next the wall is removed and the size of the simulated space increased by a factor of two to $1800 \times 2800$ and $2800 \times 4200$ nodes, respectively. In the first simulation, the signal reflected from the wall $x_{f}$ is measured at a receiver point located at the same distance from the centre of the investigated boundary as the source position, as illustrated 
in Figure 3(a). This signal inevitably also contains the 'direct sound'. In the second simulation [see Figure 3(b)], two signals are measured, namely at the same receiver position (this gives the direct sound $x_{d}$ ) and at the image position of the receiver; the latter being the freefield wave signal $x_{i}$ which arrives after travelling the same distance as the reflected signal. After the simulations, the direct sound signal is subtracted from the reflected signal obtained with the first simulation to give the isolated reflected signal $x_{r}=x_{f}-x_{d}$.

In order to analyse phase aspects of numerical boundary models, a time domain comparison of the reflected signals from the numerical experiments with the theoretical reflection signals is presented in subsequent sections. For frequency-independent boundaries, the theoretical time domain signal, which is used as a reference, is obtained by multiplying the freefield signal $x_{i}$ with the theoretical reflectance given by Equation (15). For frequency-dependent boundaries, the ideal time domain signal is obtained as a time domain convolution of the freefield signal $x_{i}$ and the signal obtained from the inverse Laplace transform of the theoretical reflectance given by Equation (15). The choice of the Laplace transform was made to avoid the characteristic ripples caused by the inverse Fourier transforms. In addition, all signals for time-domain plotting were filtered using a 41-tap low pass FIR filter in order to mask the (small) effects of truncating the dispersive tail.

The performance of boundary models is further illustrated through the comparison of the numerical and theoretical reflectance amplitude in the frequency domain. The experimentally determined numerical reflectance is calculated as the frequency-domain deconvolution of $x_{r}$ and $x_{i}$, while the theoretical reflectance given by Equation (15) is used as a reference. Furthermore, all measured signals were windowed with the use of the right half of the Hanning window before applying Fourier transforms, in order to reduce signal truncation effects.

The values of parameters related to specific resistance, mass, and spring were primarily chosen to illustrate the numerical performance of the boundary models presented in this paper; they are not based on experimental data.

To empirically confirm the stability of boundaries and corners, a set of very long simulations of small enclosures was executed, using either the standard leapfrog scheme or the interpolated DWM scheme for implementation of the room interior. Each boundary of the rectangular/cubic acoustic space consisted of 10 nodes only in order to bring about a maximum number of reflections during the $20 \mathrm{sec}-$ onds long simulation. No unstable growth was detected in any of these experiments, which confirms the stability proof provided in Section 7.2.

\subsection{D Frequency-Independent Boundary}

The results for analysis in time and frequency domains were obtained using the simulation setup described in Section 8.1 with the ADI implementation of the interior of the room; whereas in subsection on NBA, a standard rectilinear scheme was applied for the room interior.

\subsubsection{Time domain analysis}

The comparison of the reflected signals for the 2D frequency-independent model and the 1D models with two Courant numbers is illustrated in Figure 4. The 2D model matches exactly the expected signal in both time and amplitude. Conversely, time domain analysis of both 1D boundary models indicates that a phase shift problem is introduced. The plots show that for either choice of $\lambda$ there is only one incident angle for which the $1 \mathrm{D}$ boundary yields a correct phase $\left(\theta=45^{\circ}\right.$ for $\lambda=1$ and $\theta=0^{\circ}$ for $\left.\lambda=\frac{1}{\sqrt{2}}\right)$. For all other incidences, a systematic phase error results, while the amplitude generally exceeds that of the ideal theoretical reference signal.

\subsubsection{Reflectance magnitude analysis}

Figure 5 shows the reflectance obtained from numerical experiments (solid lines) plotted against the theoretical reflection (dotted lines). Concerning the 2D model, the theoretical reflection factor is very well matched for up to half the Nyquist frequency (denoted in all figures as $0.25 f_{s}$ ) even for very low values of the reflection coefficient and high angles of incidence. In particular, the numerical reflectance adheres exactly to the theoretical value at low frequencies, which is a desired property in FD modelling. Conversely, both 1D models do not adhere to the theoretical reflection factor for the majority of angles of incidence and reflection factors. In general, both 1D models approximate the magnitude incorrectly at high angles of incidence, e.g. the amplitude deviation at $\theta=75^{\circ}$ is enormous. This misalignment is particularly severe for low values of the reflection coefficient in the 1D model with $\lambda=1$ (used in DWM), which has a general tendency to outstrip the expected amplitude at all incidences, and not even approximates theory at and around $\omega=0$. This means that with such a boundary, there is no scope for improvement in accuracy by increasing the sample rate. Note that the jumps near $\omega=0$ in the experimentally obtained reflectance - particularly visible for 1D models with highest impedance value - are artefacts due to the wavefront not being perfectly flat.

\subsubsection{Numerical Boundary Analysis}

Figure 6 shows an almost perfect match for $\theta=0^{\circ}$ between the NBA and the experiments. As will be shown for frequency-dependent boundaries, similarly good matches 


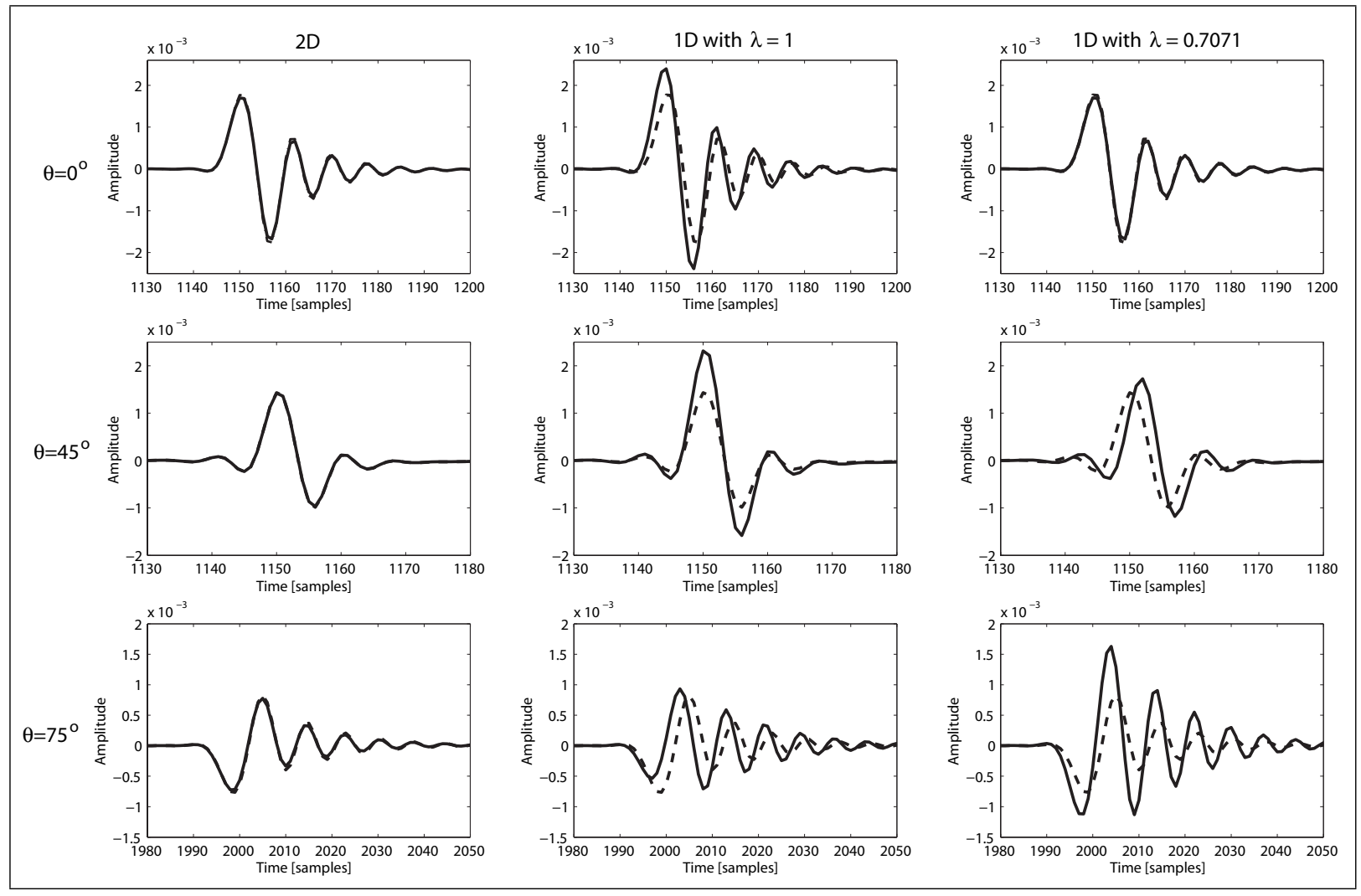

Figure 4: Reflected signal (solid line) plotted against the theoretical reference signal (dashed line) for the following angles of incidence $\theta=0^{\circ}, 45^{\circ}$ and $75^{\circ}$, and a specific wall impedance $\xi_{w}=9$.

result for all the other angles of incidence and impedance values. This important result validates both methods as tools for analysing numerical boundary formulations. In particular it enables to predict the numerical reflectance for leapfrog rectilinear scheme room interior implementations.

In order to visualize the discrepancy between theoretical and numerical reflectance in a more generalized manner, one can define the following numerical reflectance error

$$
\epsilon(\theta, \omega)=\sqrt{R(\theta, \omega)^{2}-\widehat{R}(\theta, \omega)^{2}},
$$

which takes into account both amplitude and phase deviations, because $\epsilon(\theta, \omega)$ represents the distance between the complex numbers $R(\theta, \omega)$ and $\widehat{R}(\theta, \omega)$. As illustrated in Figure 7, the numerical error is most severe at high frequencies for angles of incidence in nearly axial directions, which coincides with the fact that the numerical dispersion of the $2 \mathrm{D}$ rectilinear scheme is the strongest in axial directions. On the other hand, the horizontal white band indicates very low numerical error at and near $\theta=45^{\circ}$, which coincides with the fact the the leapfrog scheme is dispersionless in diagonal directions.

\subsection{D Frequency-Dependent Boundaries}

\subsubsection{Time domain analysis}

The phase analysis of frequency-dependent boundary model is limited to one plot, since similar results were obtained for all other angles; for more results see [22]. As depicted in Figure 8, the complex impedance boundary model preserves the phase and the amplitude is very well matched even at very high angles of incidence. Such a correct phase characteristic of this model is essentially down to the use of symmetric approximations only, namely centered difference operators and bilinear transforms which have excellent phase properties.

\subsubsection{Reflectance magnitude analysis}

The numerical reflectance amplitude is illustrated in Figure 9 for two example impedance values and different angles of incidence; for more results see [22]. The theoretical reflectance is matched well in general for up to a quarter of the sample rate for various complex impedances and all angles of incidence. As with frequency-independent boundaries, the numerical reflectance adheres perfectly at low frequencies. 


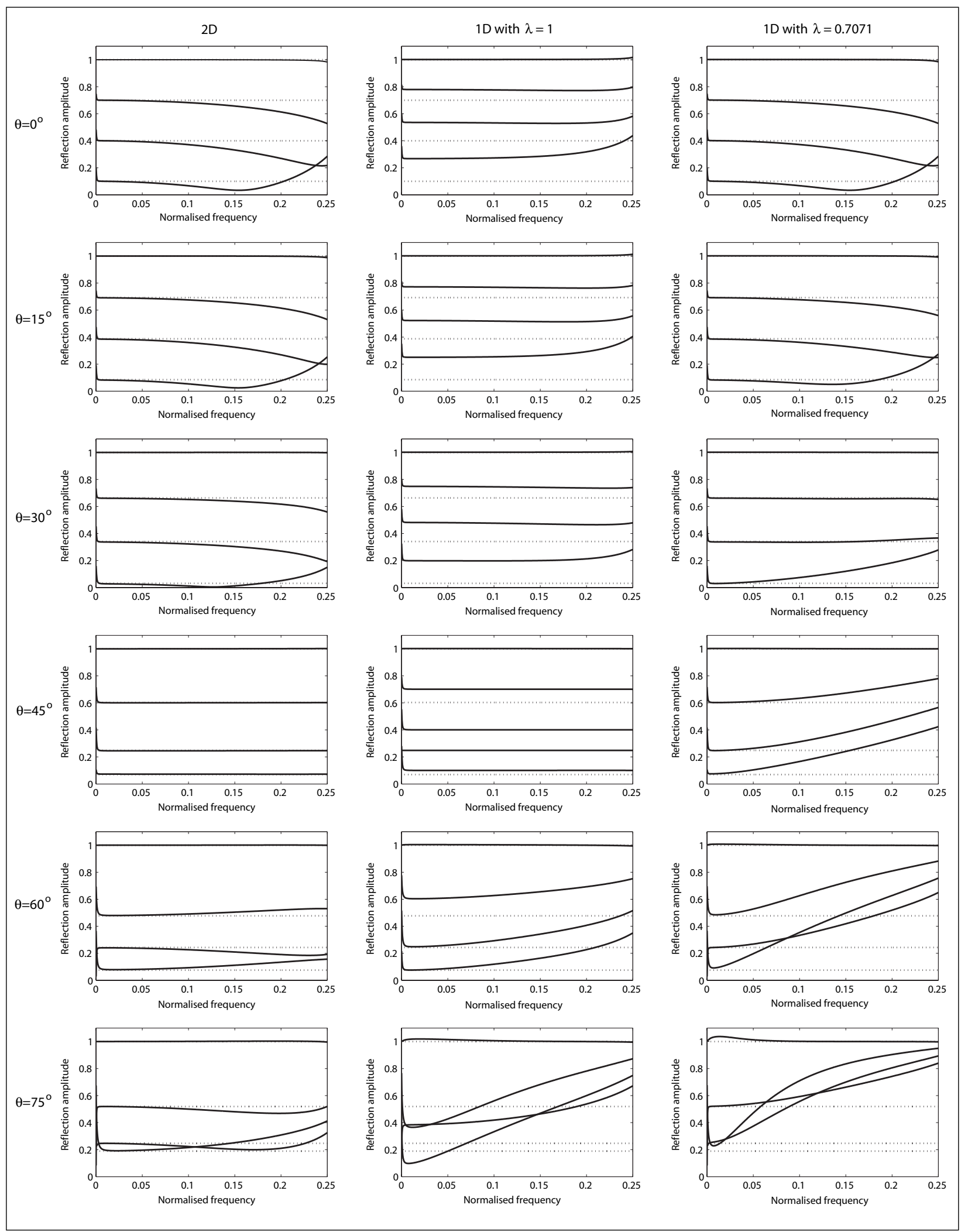

Figure 5: Numerical reflectance amplitude (solid lines) plotted against theoretical reflectance amplitude (dotted lines) for the following specific acoustic impedances $\xi_{w}=\frac{11}{9}, \frac{7}{3}, \frac{17}{3}, 10000$ and angles of incidence $\theta=0^{\circ}, 15^{\circ}, 30^{\circ}, 45^{\circ}, 60^{\circ}, 75^{\circ}$. 


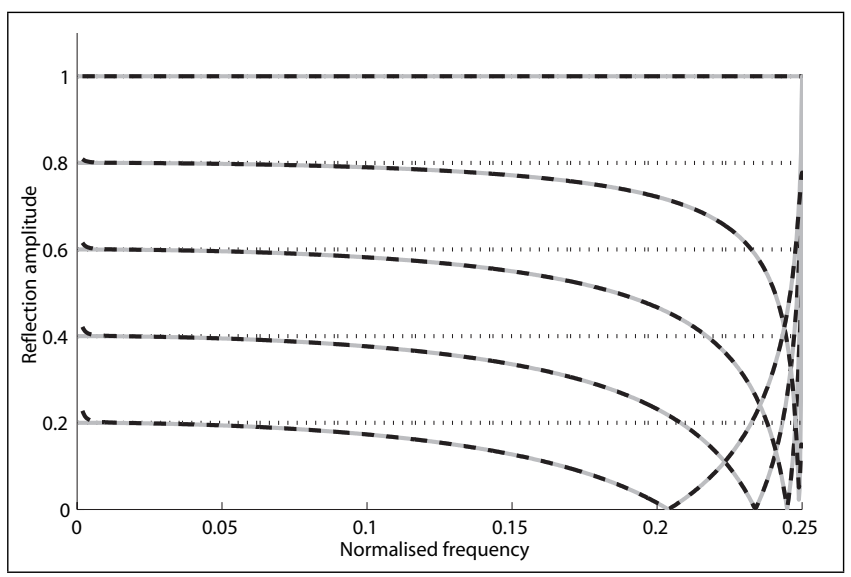

Figure 6: Numerical reflectance amplitude of a 2D boundary formulation for $\xi_{w}=\frac{3}{2}, \frac{7}{3}, 4,9,10000$ and normal incidence $\theta=0^{\circ}$. Dashed lines denote the reflectance (obtained from numerical measurements using the standard leapfrog scheme for implementation of the room interior), dotted lines denote the theoretical reflectance, and grey solid lines denote numerical reflectance obtained from the numerical boundary analysis (NBA).

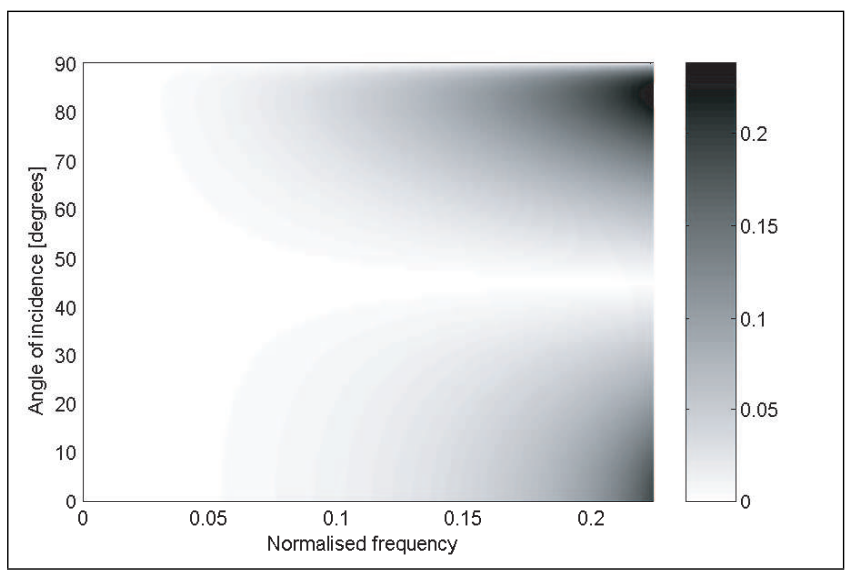

Figure 7: Numerical reflectance error of a 2D boundary model for a specific wall impedance $\xi_{w}=6$.

The reader is reminded here that the LRS theory assumes plane waves, while in the experiments a spherical wave is excited. Even though the curvature of the wavefront becomes increasingly plane with travelling distance, minor discrepancies remain, partly also due to the mesh-induced dispersion error (this is particularly so when the leapfrog scheme is used). This results in small jumps near $\omega=0$, that are particularly visible at very high angles of incidence. However it can be seen from the NBA plots that such sudden outstrips near $\omega=0$ do in fact not occur for the proposed boundary models.

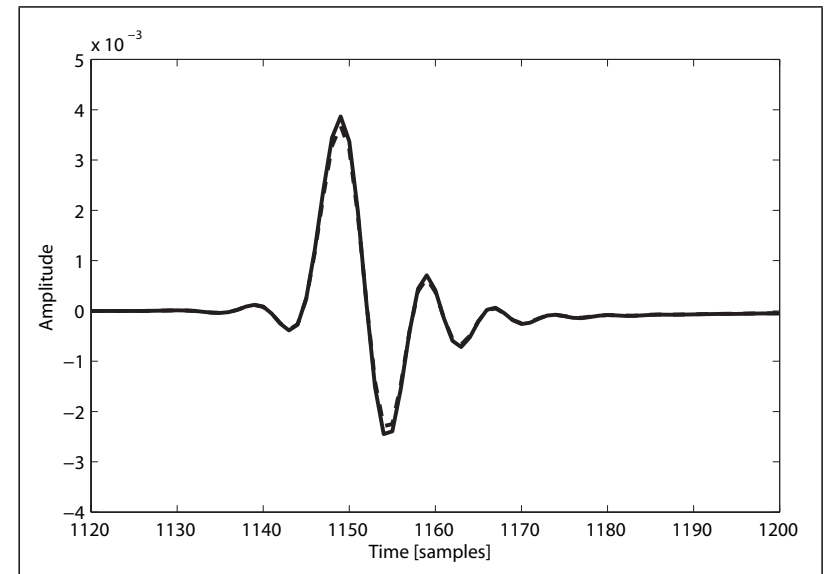

Figure 8: Reflected signal (solid line) compared to the theoretical reference signal (dashed line) for the frequencydependent formulation, where $\theta=60^{\circ}$ and $a_{R}=9$, $a_{K}=0.5$, and $a_{M}=10$.

Figure 9 shows a perfect match between the measured and predicted reflectance for $\theta=45^{\circ}$, which is the angle of incidence for which neither the rectilinear scheme used in the NBA derivations nor the compact implicit scheme used for the room interior in numerical tests exhibit any numerical error. There is also a good match for all possible angles of incidence and impedance values at low frequencies, where almost no numerical error occurs. The difference between the simulated and predicted numerical reflectance at high frequencies is due only to using a different scheme for the room interior. A closer match of the reflectance obtained from experiments than from NBA indicates that, despite the scheme discontinuity, a combination of the ADI method with the proposed boundary model actually leads to a numerical reflectance that is closer to theory than with using the leapfrog scheme consistently.

In addition, we have tested the application of the backward Euler method instead of the bilinear transform for the numerical integration and differentiation associated with the spring and mass boundary parameters, respectively. However, using such asymmetric approximations resulted in a less accurate reflectance phase and amplitude, even though the reflectance was correct in its dependence on the angle of incidence.

For comparison, we also have tested the boundary model proposed by Botteldooren in [5], now using Yee's classical staggered grid formulation to implement the interior. An example result of which is illustrated in Figure 10. Despite the fact that the backward Euler method is used for both differentiation and integration, Botteldooren's model for the staggered grid performs almost equally well to the model presented in this paper for the unstaggered grid, and yields results that are correct both in phase and amplitude. A pos- 

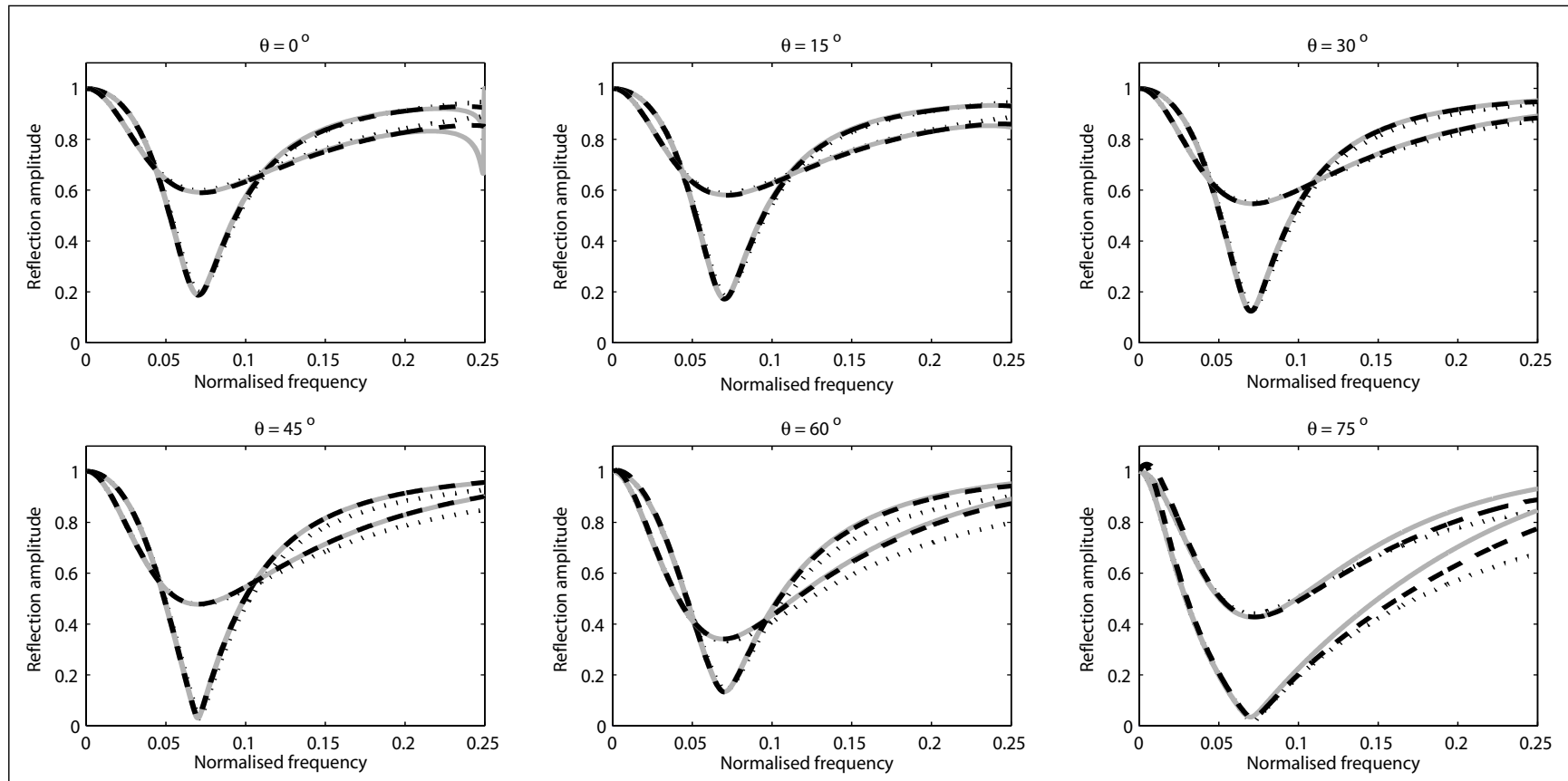

Figure 9: Numerical reflectance of a 2D complex impedance boundary for the following values of parameters $a_{R}=\frac{3}{2}, 4, a_{K}=0.5, a_{M}=$ 10 and angles of incidence $\theta=0^{\circ}, 15^{\circ}, 30^{\circ}, 45^{\circ}, 60^{\circ}, 75^{\circ}$. Dashed lines denote the reflectance obtained from numerical measurements, dotted lines denote the theoretical reflectance, and grey solid lines represent numerical reflectance obtained from NBA.

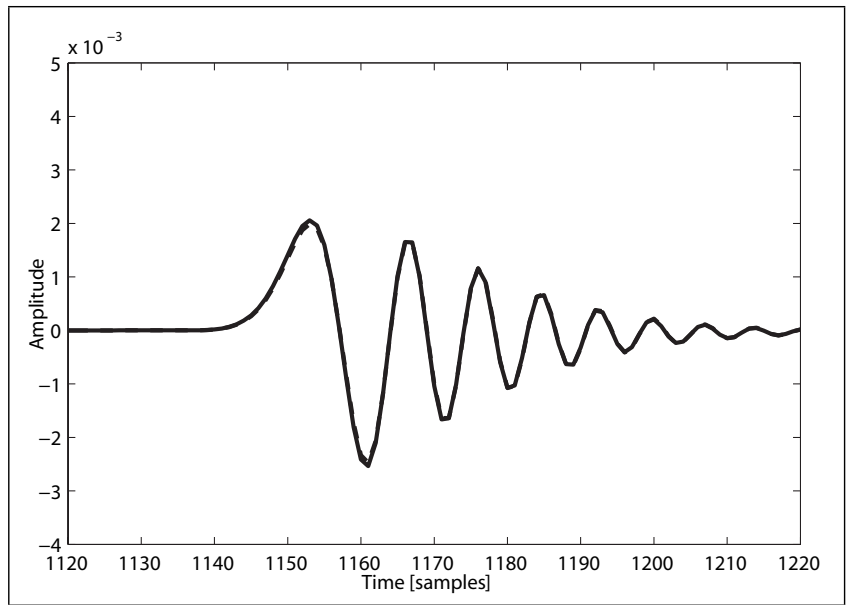

Figure 10: Reflected signal (solid line) plotted against the theoretical reference signal (dashed line) for the 2D complex impedance boundary model proposed by Botteldooren in [5] at the angle of incidence $\theta=60^{\circ}$, where $a_{R}=9, a_{K}=0.5$, and $a_{M}=10$.

sible explanation could be that the linear interpolation that is applied in his method compensates for the asymmetry of the Backward Euler method.

\subsection{D boundary model}

The reflectance magnitude of the 3D frequency-dependent model obtained with NBA for various impedances, azimuth angles, and a constant elevation angle $\phi=60^{\circ}$ is depicted in Figure 11 up to the cut-off frequency in the axial direction (i.e. up to $0.196 f_{s}$ ). These results indicate that the proposed boundary model yields highly accurate approximation of the expected reflectance even at high incidences, for both azimuth and elevation angles, and low impedance values.

The numerical reflectance error according to Equation (73) is illustrated in Figure 12 for a specific acoustic impedance 6 , a constant azimuth angle of incidence $\theta=$ $45^{\circ}$, and varying elevation angle $\phi$. As in the 2D case, the wave equation is dispersionless at diagonal directions, which occurs for $\theta=45^{\circ}$ and $\phi=\arcsin (1 / \sqrt{3}) \approx 35.3^{\circ}$. As can be seen from Figure 12, the numerical reflectance error also vanishes in this direction. Hence it can be concluded that the numerical error generally manifests itself in the same way for the boundary and the interior.

\section{Conclusions}

In this paper, we have proposed a new method for constructing numerical formulations of locally reacting surfaces that can be applied to finite difference time domain modelling 

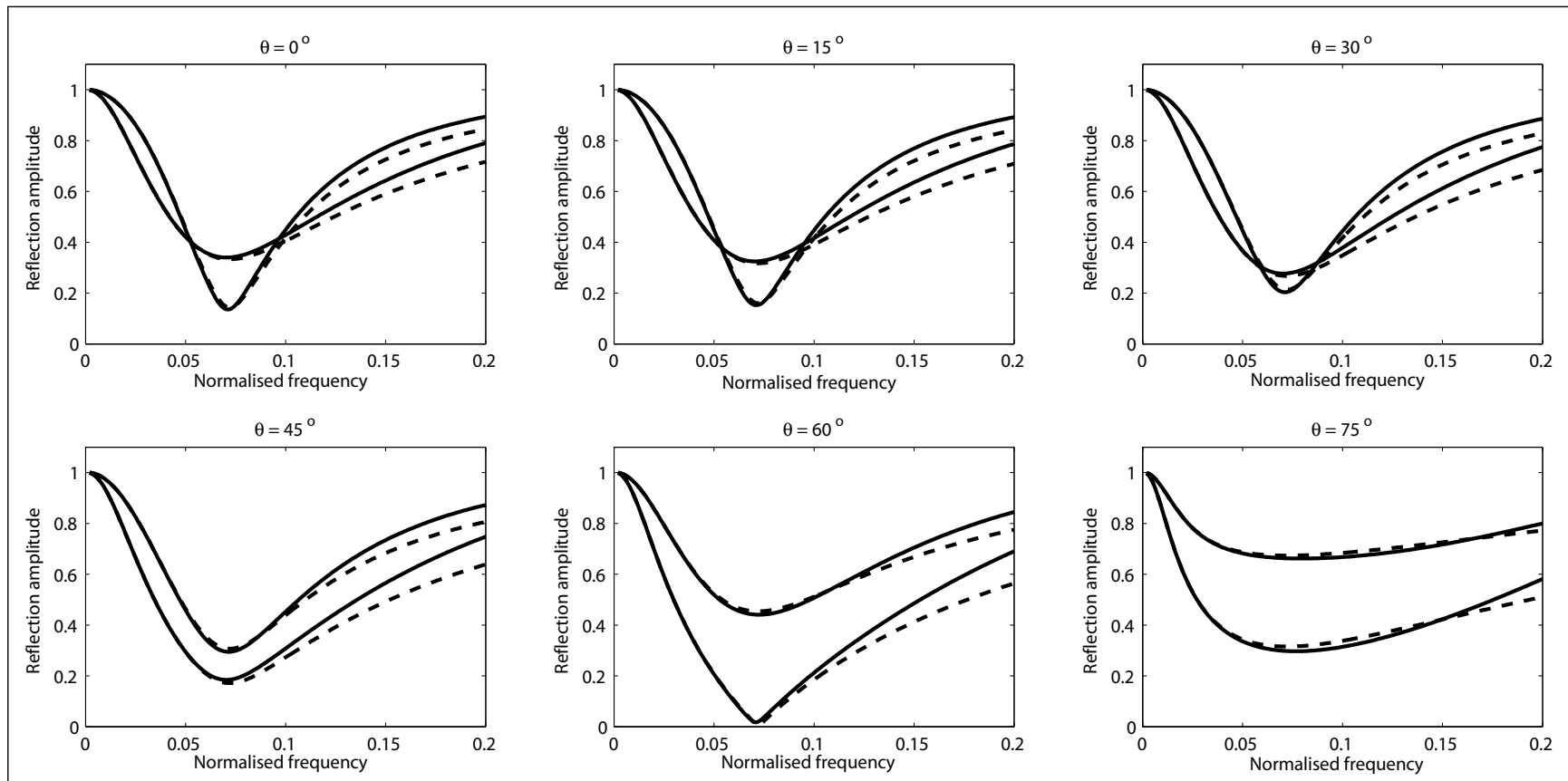

Figure 11: Numerical reflectance amplitude of a 3D complex impedance boundary for the following values of parameters $a_{R}=\frac{3}{2}, 4, a_{K}=0.5, a_{M}=10$, the elevation angle $\phi=60^{\circ}$, and azimuth angles $\theta=0^{\circ}, 15^{\circ}, 30^{\circ}, 45^{\circ}, 60^{\circ}, 75^{\circ}$. Numerical reflectance obtained from the analytic evaluation method (solid lines) is plotted against theoretical values (dashed lines) up to $0.196 f_{s}$.

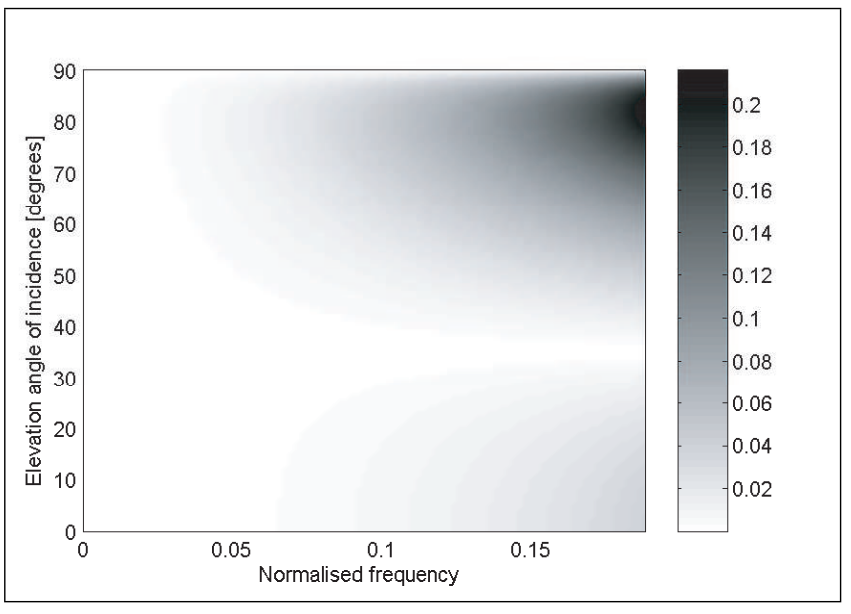

Figure 12: Numerical reflectance error of a 3D boundary model for a real specific wall impedance $\xi_{w}=6$ and azimuth angle $\theta=45^{\circ}$.

of room acoustics. Novel formulations were presented for simple frequency-dependent boundaries in which the wall is characterized by a complex impedance expression that incorporates linear resistance, inertia, and restoring forces. A good match with theory was demonstrated for all angles of incidence and boundary parameters. Generally, these boundary models yield accurate results both in reflectance phase and amplitude for frequencies up to near the axial cut-off frequency $\left(0.25 f_{s}\right.$ for $2 \mathrm{D}$ case and $0.196 f_{s}$ for $3 \mathrm{D}$ systems). As such, the proposed formulations represent a significant improvement over the $1 \mathrm{D}$ boundary formulations used in digital waveguide mesh modelling. It has been shown that for frequency-independent boundaries, the 1D formulation structurally exhibits a phase error, which implies that in a simulation of an enclosed space, the boundaries are effectively positioned closer to each other than intended. In addition, the reflectance magnitude of 1D boundary models systematically exceeds the theoretical reflectance value, particularly so for high angles of incidence and low impedance values. While our analysis has focused on 2D simulations, these inaccuracies are even more pronounced in 3D simulations, due to larger numerical errors and the increased range of possible angles of incidence. The advantages of the proposed 2D/3D approach over the 1D approach also apply to frequency-dependent boundaries. That is, the boundary filter formulation using $\mathrm{KW}$ pipes, which until now is the main frequency-dependent boundary formulation available for unstaggered grid FDTD and K-DWM simulations, is based on exactly the same principles as its frequency-independent counterpart, and in fact reduces to that when the boundary filter is set to a constant.

The work described in this paper also represents an im- 
provement to Botteldooren's method, in that the use of symmetric finite difference operators leads to a boundary formulation that does not introduce an additional numerical stability condition. Hence, unlike with Botteldooren's formulation for the staggered grid, which uses asymmetrical operators, the model presented here for the standard leapfrog scheme allows for a completely free choice of boundary parameters.

In addition, we have introduced a novel analytic method for exact prediction of the numerical reflectance of 2D/3D boundary models. This numerical boundary analysis (NBA) method was validated by a precise match with experimental results. As such, the NBA provides a fast and precise tool for evaluating multi-dimensional boundary formulations, removing the need for carrying out elaborate numerical experiments that are time consuming, require enormous computer memory, and are prone to small artefacts due to violation of the assumption of plane waves.

In addition, the $2 \mathrm{D} / 3 \mathrm{D}$ boundary formulation allows a proper formulation of corner and edge nodes. In the literature, this problem appears to have never been addressed in detail in the context of DWM and FDTD methods applied to room acoustics.

The boundary models derived in this paper are intended for FDTD room acoustic simulations with the use of the standard leapfrog scheme. The consistency in scheme applied for the room interior and at the boundary implies that analytic techniques can be used to unambiguously predict the performance of both the medium and the boundary. Furthermore, the stability of the whole simulation is quaranteed. The same applies to the mathematically equivalent rectangular DWM implemented using Kirchhoff variables, with which the presented boundary models can be combined.

Alternative approach is to combine the boundary for the standard leapfrog scheme with other schemes based on the rectilinear mesh, e.g. the ADI scheme or the interpolated rectilinear mesh. For instance, in this paper we used the ADI scheme to obtain accurate reflectance results. Nevertherless, due to scheme discontinuity at the boundary, the stability of such a simulation cannot be quaranteed. Consequently, such a combination applied to modelling a complex-shaped acoustic enclosure is not recommended.

[Maarten, I think this goes out - In such a scenario, the NBA does not exactly predict the numerical reflectance, but any deviations occur only at very high frequencies. Interestingly, the scheme discontinuity introduced by using an ADI scheme for the interior nodes actually helps reducing the numerical boundary error.]

Finally, as a reference to possible future work on this topic, we envisage that the boundary formulations for other finite difference schemes (e.g. the interpolated finite differnce mesh) and mesh topologies (e.g. the triangular mesh) may be derived in a similar way, by combining the boundary conditions presented in this paper with the relevant discrete wave equation for that particular mesh topology. Using this procedure, the resulting formulation should preserve the desired physicality as long as the boundary condition is applied across the boundary in the direction normal to the boundary.

\section{References}

[1] M. Vörlander, "International round robin on room acoustical computer simulations," Proc. Int. Congress on Acoustics, pp. 689-692, June 1995, Trondheim, Norway.

[2] U.M. Svensson, "Modelling acoustic spaces for audio virtual reality," Workshop on Model based Proc. and Coding of Audio (MPCA), pp. 109-116, November 2002, Leuven, Belgium.

[3] L. Savioja and V. Välimäki, "Reducing the dispersion error in the digital waveguide mesh using interpolation and frequency warping techniques," IEEE Trans. Speech and Audio Processing, vol. 8, pp. 184-194, March 2000.

[4] K. Kowalczyk and M. van Walstijn, "On-line simulation of 2D resonators with reduced dispersion error using compact implicit finite difference methods," Proc. IEEE Int. Conf. Acoustics, Speech and Signal Processing (ICASSP), pp. 285-288, April 2007, Honolulu, Hawaii.

[5] D. Botteldooren, "Finite-difference time-domain simulation of lowfrequency room acoustic problems," J. Acoust. Soc. Am., vol. 98, no. 6, pp. 3302-3308, 1995.

[6] S.van Duyne and J.O. Smith, "The 2-D digital waveguide mesh," Proc. IEEE Workshop on Applications of Signal Processing to Audio and Acoustics, October 1993, Mohonk, NY.

[7] M. Karjalainen and C. Erkut, "Digital waveguide vs. finite difference schemes: Equivalence and mixed modeling," EURASIP J. Applied Signal Processing, , no. 7, pp. 978-989, June 2004.

[8] A. Kelloniemi, L. Savioja, and V. Välimäki, "Spatial filter-based absorbing boundary for the 2-D digital waveguide mesh," IEEE Signal Processing Letters, vol. 12, no. 2, pp. 126-129, February 2005.

[9] L. Savioja, T. Rinne, and T. Takala, "Simulation of room acoustics with a 3-D finite difference mesh," Proc. Int. Computer Music Conf. (ICMC), pp. 463-466, September 1994, Aarhus, Denmark.

[10] J. Huopaniemi, L. Savioja, and M. Karjalainen, "Modeling of reflections and air absorption in acoustical spaces - a digital filter design approach," Proc. IEEE Workshop on Appl. of Signal Processing to Audio and Acoustics (WASPAA), pp. 1-4, October 1997, New Palz, NY.

[11] A. Kelloniemi, "Frequency-dependent boundary condition for the 3D digital waveguide mesh," Proc. Int. Conf. Digital Audio Effects (DAFx'06), pp. 161-164, September 2006, Montreal, Canada.

[12] D.T. Murphy and M. Beeson, "The KW-boundary hybrid digital waveguide mesh for room acoustics applications," IEEE Transactions on Speech and Audio Processing, vol. 12, no. 2, pp. 552-564, 2007.

[13] K. Kowalczyk and M. van Walstijn, "Formulation of a locally reacting wall in finite difference modelling of acoustic spaces," Proc. Int. Symp. Room Acoustics (ISRA), pp. 1-6, September 2007, Seville, Spain.

[14] H. Kuttruff, Room Acoustics, Applied Science Publishers Ltd, London, 1973.

[15] S. Bilbao, Wave and Scattering Methods for Numerical Simulation, John Wiley \& Sons, London, 2004. 
[16] A. Taflove and S.C. Hagness, Computational Electrodynamics: The Finite-Difference Time-Domain Method, 2nd ed., Artech House, Norwood, MA, 2000.

[17] J.W. Thomas, Numerical Partial Differential Equations: Finite Difference Methods, Springer-Verlag, New York, 1998.

[18] L. Savioja, M. Karjalainen, and T. Takala, "DSP formulation of a finite difference method for room acoustics simulation," Proc. IEEE Nordic Signal Processing Symp. (NORSIG'96), vol. 2, pp. 455-458, September 1996, Espoo, Finland.

[19] D.T. Murphy and J. Mullen, "Digital waveguide mesh modelling of room acoustics: Improved anechoic boundaries," Proc. Int. Conf. Digital Audio Effects (DAFx'02), pp. 163-168, September 2002, Hamburg, Germany.

[20] B. Gustafsson, H.-O. Kreiss, and A. Sundström, Time Dependent Problems and Difference Methods, John Wiley \& Sons, New York, 1995.

[21] J.O. Smith, Physical Audio Signal Processing for Virtual Musical Instruments and Digital Audio Effects, W3K Publishing, http://books.wk3.org, August 2006.

[22] K. Kowalczyk and M. van Walstijn, "Virtual room acoustics using finite difference methods. How to model and analyse frequencydependent boundaries?," Proc. IEEE Int. Symp. Communications, Control and Signal Processing (ISCCSP), pp. 1504-1509, March 2008, Malta. 